\title{
EQUIVALENT CONDITIONS FOR STABILIZABILITY OF INFINITE-DIMENSIONAL SYSTEMS WITH ADMISSIBLE CONTROL OPERATORS*
}

\author{
BIRGIT $\mathrm{JACOB}^{\dagger}$ AND HANS ZWART
}

\begin{abstract}
In this paper we study the optimizability of infinite-dimensional systems with admissible control operators. We show that under a weak condition such a system is optimizable if and only if the system can be split into an exponentially stable subsystem and an unstable subsystem that is exactly controllable in finite time. The state space of the unstable subsystem equals the span of all unstable (generalized) eigenvectors of the original system. This subsystem can be infinite-dimensional. Furthermore, the unstable poles satisfy a summability condition. The state space of the exponentially stable subsystem is given by all vectors for which the action of the original $C_{0}$-semigroup is stable.
\end{abstract}

Key words. infinite-dimensional systems, stabilizability, optimizability, controllability

AMS subject classifications. 93C25, 93D15, 93B05

PII. S036301299833344X

1. Introduction. This paper is concerned with the stabilization of infinitedimensional systems with a finite-dimensional input space described by the following abstract differential equation:

$$
\begin{aligned}
\dot{x}(t) & =A x(t)+B u(t), \quad t \geq 0 \\
x(0) & =x_{0},
\end{aligned}
$$

where $A$ is the infinitesimal generator of the $C_{0}$-semigroup $T(t)$ on the separable Hilbert space $H$ and $B$ is the control operator.

Since the early days of infinite-dimensional systems theory, researchers have tried to characterize conditions under which (1) is stabilizable; see, e.g., the survey articles by Pritchard and Zabczyk [23] and by Russell [29]. In the early 1970s Datko [4] found a characterization in terms of the cost functional

$$
J\left(x_{0}, u\right):=\int_{0}^{\infty}\|x(t)\|^{2}+\|u(t)\|^{2} d t
$$

He proved that if $B$ is a bounded operator, then there exists a bounded feedback $F$ such that $A+B F$ generates an exponentially stable semigroup if and only if for every $x_{0} \in H$ there exists an input $u$ such that $J\left(x_{0}, u\right)<\infty$.

It was only in the mid-1980s that for the class of bounded control operators other necessary and sufficient conditions were found; see Desch and Schappacher [5], Jacobson and Nett [17], and Nefedov and Sholokhovich [20]. Independently of each other, they showed the following result. Here $\mathcal{L}\left(X_{1}, X_{2}\right)$ denotes the class of linear, bounded operators from $X_{1}$ to $X_{2}$.

\footnotetext{
*Received by the editors January 30, 1998; accepted for publication (in revised form) October 22, 1998; published electronically July 27, 1999. This research was supported by the Human Capital and Mobility program (project number CHRX-CT 93-0402) and Volkswagen-Stiftung (RiP-program at Oberwolfach).

http://www.siam.org/journals/sicon/37-5/33344.html

†School of Mathematics, University of Leeds, Leeds LS2 9JT, UK (birgit@amsta.leeds.ac.uk).

$\ddagger$ Faculty of Mathematical Sciences, University of Twente, P. O. Box 217, 7500 AE Enschede, The Netherlands (H.J.Zwart@math.utwente.nl).
} 
Theorem 1.1. Consider the system (1) with $B \in \mathcal{L}\left(\mathbb{C}^{m}, H\right)$. Then the following conditions are equivalent.

1. There exists an $F \in \mathcal{L}\left(H, \mathbb{C}^{m}\right)$ such that $A+B F$ generates an exponentially stable $C_{0}$-semigroup.

2. It is possible to split the state space as $H=H_{u} \oplus H_{s}$ such that $\left.T(t)\right|_{H_{s}}$ is exponentially stable, $H_{u}$ is finite-dimensional and the restriction of (1) to $H_{u}$ is exactly controllable in finite time.

Looking at the proof of this theorem, we observe that from the fact that $B$ and $F$ are bounded, finite-rank operators it was obtained that there are only finitely many unstable eigenvalues. Furthermore, $H_{u}$ is the span over the eigenspaces corresponding to these eigenvalues.

In the eighties the first results for unbounded control operators were found. In general, the class of unbounded control operators consists of those operators $B$ for which

$$
\int_{0}^{t_{1}} T\left(t_{1}-\rho\right) B u(\rho) d \rho
$$

defines a bounded linear operator from $\boldsymbol{L}_{2}\left(0, t_{1} ; \mathbb{C}^{m}\right)$ to $H$. Following Weiss [32], we call $B$ an admissible control operator for $T(t)$. If $B$ is such an admissible control operator for $T(t)$, then for every $x_{0}$ and for every $u \in \boldsymbol{L}_{2}^{\text {loc }}\left(0, \infty ; \mathbb{C}^{m}\right),(1)$ has a unique solution $x(\cdot)$ which lies in $H$ and is continuous; see Weiss [33].

Flandoli, Lasiecka, and Triggiani [8] extended the result of Datko to the class of admissible control operators for $T(t)$. Among others, they showed the following.

TheOREM 1.2. Consider the system (1), where the operator $B$ is an admissible control operator for $T(t)$. Then the following conditions are equivalent.

1. System (1) is optimizable; i.e., for every element $x_{0} \in H$ there exists an input $u \in \boldsymbol{L}_{2}\left(0, \infty ; \mathbb{C}^{m}\right)$ such that the cost functional $J\left(x_{0}, u\right)$, given by $(2)$, is finite.

2. There exist an exponentially stable semigroup $T_{F}(t)$ with infinitesimal generator $A_{F}: D\left(A_{F}\right) \rightarrow H$, and an $F \in \mathcal{L}\left(D\left(A_{F}\right), \mathbb{C}^{m}\right)$ such that

(a) $F T_{F}(\cdot): D\left(A_{F}\right) \rightarrow \boldsymbol{L}_{2}\left(0, \infty ; \mathbb{C}^{m}\right)$ extends to a bounded linear operator from $\mathrm{H}$ to $\boldsymbol{L}_{2}\left(0, \infty ; \mathbb{C}^{m}\right)$;

(b) For every $t>0$ and $x_{0} \in H$ there holds

$$
T_{F}(t) x_{0}=T(t) x_{0}+\int_{0}^{t} T(t-\rho) B F T_{F}(\rho) x_{0} d \rho .
$$

The extension of Theorem 1.1 for the case that $B$ is an admissible control operator for $T(t)$ and $F$ is a bounded operator can be found in Rebarber [25]. The aim of this paper is to show that Theorem 1.1 can be extended to admissible control operators for $T(t)$ and to feedback operators satisfying condition 2 of Theorem 1.2. The implication part 2 to part 1 is relatively easy, so we will concentrate on the other direction.

Before we summarize our results, we have to introduce some notation. We say that the generator $A$ of a $C_{0}$-semigroup satisfies the spectrum decomposition assumption (SDA) at $g, g \in \mathbb{R}$, if there exist real numbers $g_{1}<g<g_{2}$ such that there is no spectrum of $A$ with real part between $g_{1}$ and $g_{2}$. In particular, we say that $A$ satisfies the SDA if $A$ satisfies SDA at 0 . Hence the SDA states that the spectrum can be split into a "stable" and an "unstable" part. The main result that we prove in this paper is the following. 
TheOREM 1.3. Consider the system (1), where the operator $B$ is an admissible finite-rank control operator for $T(t)$. If the system is optimizable and A satisfies the $S D A$, then it is possible to split the state space as $H=H_{s} \oplus H_{u}$, where

$$
\begin{aligned}
& H_{s}=\left\{x_{0} \in H \mid T(t) x_{0} \in \boldsymbol{L}_{2}(0, \infty ; H)\right\}, \\
& H_{u}=\frac{\operatorname{span}_{\lambda \in \sigma(A) \text { with } \operatorname{Re}(\lambda) \geq 0} P_{\lambda} H}{} .
\end{aligned}
$$

Here $P_{\lambda} H$ denotes the spectral subspace corresponding to the eigenvalue $\lambda$.

Moreover, $H_{s}$ as well as $H_{u}$ is a $T(t)$-invariant subspace, $\left.T(t)\right|_{H_{s}}$ is exponentially stable, $\left.T(t)\right|_{H_{u}}$ is a group and the restriction of (1) to $H_{u}$ is exactly controllable in finite time.

A result similar to those of Theorem 1.3 holds if the system $(A+g I, B)$ is optimizable and if $A+g I$ satisfies the SDA. So one sees that the only difference between bounded and unbounded control operators lies in the fact that $H_{u}$ may be infinitedimensional and that we have to assume the SDA.

One may interpret this theorem as follows. If the system is optimizable and the spectrum can be split into a stable and unstable part, then the state space can be split in the corresponding way. Note that for general infinite-dimensional systems such a splitting of the state space is not always possible, even if one can split the spectrum. There exist infinitesimal generators whose spectrum lies in the left-half plane and is bounded away from the imaginary axis, but they generate an unstable semigroup; see, e.g., Zabczyk [39].

It may seem that the spectrum decomposition assumption is very strong. However, in Rebarber and Zwart [28, Theorem 2.11] the following result can be found.

THEOREM 1.4. Assume that system (1) is optimizable where the operator $B$ is an admissible finite-rank control operator for $T(t)$. Then there exists an $\varepsilon>0$ such that all elements in the spectrum of $A$ with real part larger than $-\varepsilon$ are eigenvalues of $A$ with finite algebraic multiplicity and all accumulation points of the spectrum of $A$ have real part less than or equal to $-\varepsilon$.

Hence this result gives a necessary condition for the system (1) to be optimizable. Looking now at the class of systems that satisfies this necessary condition, i.e., in the closed right-half plane there is only point spectrum, it is much harder to find an example that does not satisfy the SDA. All delay and partial differential equations that satisfy the necessary condition on its spectrum also satisfy the SDA at $-\varepsilon$ for some $\varepsilon>0$.

We construct an example that is optimizable, but it is not possible to split the state space in a direct sum as given in Theorem 1.3. The generator does not satisfy the SDA at any negative number.

If $A$ is a Riesz-spectral operator, then we are able to prove Theorem 1.3 without assuming that $A$ satisfies SDA. The SDA at $-\varepsilon$, for some $\varepsilon>0$, will automatically be satisfied if the system is stabilizable by a bounded feedback $B F$, since then there can only be finitely many eigenvalues with real part larger than $-\varepsilon$ for some $\varepsilon>0$.

From Rebarber and Zwart [28] we have that the unstable part of the spectrum is pure point spectrum; see Theorem 1.4. Here we show an extra property concerning the distribution of this point spectrum.

THEOREM 1.5. Assume that system (1) is optimizable where the operator $B$ is an admissible finite-rank control operator for $T(t)$. Let $\left\{\lambda_{n}\right\}$ denote the set of eigenvalues with real part greater than 0 and let $m_{a}\left(\lambda_{n}, A\right)$ denote the algebraic multiplicity of $\lambda_{n}$. 
Then we have

$$
\sum_{n \in \mathbb{N}} \frac{m_{a}\left(\lambda_{n}, A\right)}{\left|\lambda_{n}\right|^{2}}<\infty .
$$

From Theorem 1.3 we obtain some consequences as easy corollaries. For instance, if the spectrum of $A$ lies in the left-half plane and is bounded away from the imaginary axis, but $A$ generates an unstable semigroup, then it is never optimizable, for any admissible finite-rank control operator for $T(t)$. Thus these systems will never be exactly controllable in finite time either. Examples of such generators can be found in, e.g., Zabczyk [39] and Greiner, Voigt, and Wolff [11]. This result can be found in [28] for the case of one-dimensional, not necessarily admissible, input operators.

Another consequence is the following. Suppose that $A$ generates a $C_{0}$-group. If (1) is exactly controllable in finite time for some $B$ where $B$ is an admissible finite-rank control operator for $T(t)$, then the (generalized) eigenfunctions of $A$ must span $H$. This follows easily from Theorem 1.3 by using the fact that $A+\gamma I$ is exactly controllable in finite time for every $\gamma \in \mathbb{R}$. Now choose $\gamma$ so large that the corresponding "stable" subspace $H_{s}$ is empty, which is possible since $A$ generates a $C_{0}$-group.

A third consequence is the following. Suppose that $A$ generates a completely unstable $C_{0}$-semigroup, that $\Sigma(A, B)$ is optimizable, and that $B$ is an admissible finite-rank control operator for $T(t)$. Then $A$ generates a $C_{0}$-group, and $\Sigma(A, B)$ is exactly controllable in finite time. This is an extension of the result found by Russell in [29]. Reversing the argument gives that the right-shift semigroup on $\boldsymbol{L}_{2}(0, \infty)$ will never be optimizable nor exactly controllable in finite time by a finite-rank admissible control operator for $T(t)$. The reason is that the right-shift semigroup is completely unstable, but it is not a $C_{0}$-group.

The organization of the paper is as follows. In the next two sections we introduce the necessary background and notation. In section 4, we show that if the state space can be split into two $T(t)$-invariant subspaces, then we can write the system (1) into two corresponding subsystems. Theorem 1.3 will be proved in section 5 , apart from the characterization of $H_{s}$ and $H_{u}$. That will be done in section 7 . Theorem 1.5 will be the subject of section 6 . Finally, in section 8 we present an example that is optimizable, but it is not possible to split the state space of this example in a direct sum as given in Theorem 1.3.

2. System description. In this section, we describe the general class of systems discussed in this paper. First we need to introduce some notation.

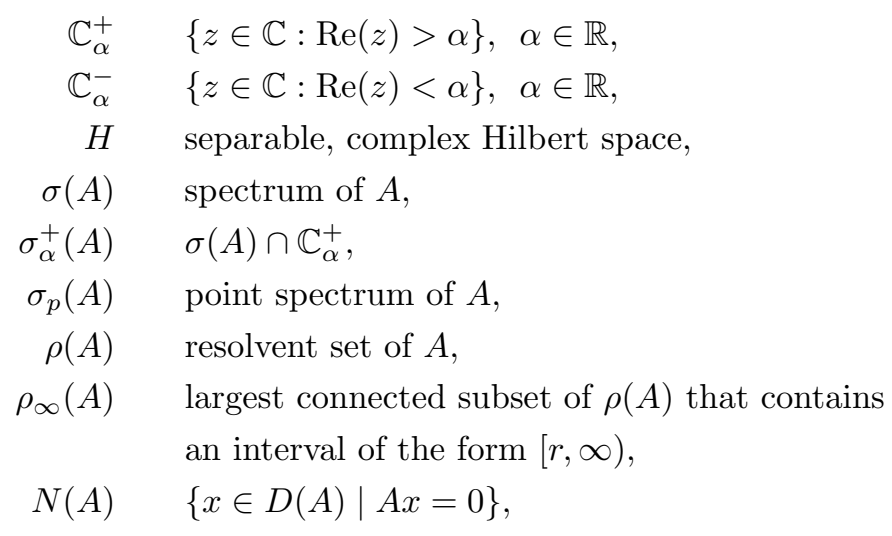




$$
\begin{aligned}
\operatorname{Im}(A) & \{x \in H \mid \exists y \in D(A): A y=x\}, \\
\boldsymbol{H}_{\infty}\left(\mathbb{C}_{\alpha}^{+} ; H\right) & \text { set of holomorphic and bounded functions } \\
& \text { from } \mathbb{C}_{\alpha}^{+} \text {to } H, \\
\boldsymbol{H}_{2}\left(\mathbb{C}_{\alpha}^{+} ; H\right) & \text { set of holomorphic functions } f: \mathbb{C}_{\alpha}^{+} \rightarrow H \text { with } \\
& \sup _{x>\alpha}\left(\int_{-\infty}^{\infty}\|f(x+i y)\|^{2} d y\right)^{1 / 2}<\infty .
\end{aligned}
$$

We deal with infinite-dimensional, time-invariant systems of the following kind:

$$
\dot{x}(t)=A x(t)+B u(t) .
$$

Here $A: D(A) \rightarrow H$ is the generator of a $C_{0}$-semigroup $T(t)$ on the separable, complex Hilbert space $H$, and $B$ will be our (unbounded) control operator. The input function $u$ is assumed to be in $\boldsymbol{L}_{2}^{\text {loc }}\left(0, \infty ; \mathbb{C}^{m}\right)$.

In order to define our class of control operators we have to introduce some notation. We define the space $H_{-1}$ to be the completion of $H$ with respect to the norm

$$
\|x\|_{-1}:=\left\|(\beta I-A)^{-1} x\right\|
$$

and the space $H_{1}$ to be $D(A)$ with the norm

$$
\|x\|_{1}:=\|(\beta I-A) x\|,
$$

where $\beta \in \rho(A)$, the resolvent set of $A$. It is easy to verify that $H_{-1}$ and $H_{1}$ do not depend on $\beta \in \rho(A)$. Moreover, $\|\cdot\|_{1}$ is equivalent to the graph norm on $D(A)$, so $H_{1}$ is complete. In Weiss [33, Remark 3.4] it is shown that $T(t)$ has a restriction to a $C_{0}$-semigroup on $H_{1}$ whose generator is the restriction of $A$ to $D(A)$, and $T(t)$ can be extended to a $C_{0}$-semigroup on $H_{-1}$ whose generator is an extension of $A$ with domain $H$. Therefore we get

$$
A \in \mathcal{L}\left(H_{1}, H\right) \quad \text { and } \quad A \in \mathcal{L}\left(H, H_{-1}\right) .
$$

$H_{-1}$ equals the dual of $D\left(A^{*}\right)$, where we have equipped $D\left(A^{*}\right)$ with the graph norm (see [33]). Following [33] we introduce admissible control operators for $T(t)$.

Definition 2.1. Let $B \in \mathcal{L}\left(\mathbb{C}^{m}, H_{-1}\right)$. For $t \geq 0$ we define the operator $\mathcal{B}_{t}$ : $\boldsymbol{L}_{2}\left(0, \infty ; \mathbb{C}^{m}\right) \rightarrow H_{-1}$ by

$$
\mathcal{B}_{t} u:=\int_{0}^{t} T(t-\rho) B u(\rho) d \rho .
$$

Then $B$ is called an admissible control operator for $T(t)$, if for some (and hence any) $t>0, \mathcal{B}_{t} \in \mathcal{L}\left(\boldsymbol{L}_{2}\left(0, \infty ; \mathbb{C}^{m}\right), H\right)$.

By a solution of (4) with initial condition $x(0)=x_{0} \in H$ we mean the function defined by the variation of parameters formula

$$
x(t)=T(t) x_{0}+\int_{0}^{t} T(t-\rho) B u(\rho) d \rho, \quad t \geq 0 .
$$

Note that the admissibility of $B$ guarantees $x(t) \in H$ for $t \geq 0$ and in [33] it is shown that $x$ is continuous. We will denote system (4) by $\Sigma(A, B)$. 
In the following the concept of admissible observation operators for $T(t)$ also will be needed (see also Weiss [34]).

Definition 2.2. Let $C \in \mathcal{L}\left(H_{1}, \mathbb{C}^{p}\right)$. Then $C$ is called an admissible observation operator for $T(t)$, if for some (and hence any) $t>0$, there is some $K>0$ such that

$$
\|C T(\cdot) x\|_{\boldsymbol{L}_{2}\left(0, t ; \mathbb{C}^{p}\right)} \leq K\|x\|, \quad x \in D(A) .
$$

If the semigroup $T(t)$ is exponentially stable, then the constant $K$ does not depend on $t \geq 0$ (see Grabowski and Callier [10]). We end this section with some simple properties of admissible control and observation operators for $T(t)$.

REMARK 2.3. $B$ is an admissible control operator for the $C_{0}$-semigroup $T_{\tau}(t)$ given by

$$
T_{\tau}(t):=e^{\tau t} T(t)
$$

where $\tau \in \mathbb{R}$ is arbitrary. Moreover, if the operator $C \in \mathcal{L}\left(H_{1}, \mathbb{C}^{p}\right)$ is an admissible observation operator for $T(t)$, then $C$ also is an admissible observation operator for $T_{\tau}(t)$.

3. Optimizability, LQ-stabilizing feedbacks, and exact controllability. In this section we introduce the notions of optimizability, LQ-stabilizing feedbacks, and exact controllability. Furthermore, we investigate relationships between these notions. With the system $\Sigma(A, B)$ we associate the cost functional

$$
J\left(x_{0}, u\right):=\int_{0}^{\infty}\|x(t)\|^{2}+\|u(t)\|^{2} d t
$$

Definition 3.1. We call the system $\Sigma(A, B)$-optimizable, $g \in \mathbb{R}$, if for every $x_{0} \in H$ there exists an input $u \in \boldsymbol{L}_{2}^{\text {loc }}\left(0, \infty ; \mathbb{C}^{m}\right)$ such that

$$
J^{g}\left(x_{0}, u\right):=\int_{0}^{\infty}\left[\left\|e^{-g t} x(t)\right\|^{2}+\left\|e^{-g t} u(t)\right\|^{2}\right] d t<\infty .
$$

In particular, we call the system $\Sigma(A, B)$ optimizable, if it is 0-optimizable.

Thus the system $\Sigma(A, B)$ is optimizable if for every $x_{0} \in H$ there exists an input $u \in \boldsymbol{L}_{2}\left(0, \infty ; \mathbb{C}^{m}\right)$ such that the cost functional $J\left(x_{0}, u\right)$ is finite. Optimizability is also known as the finite cost condition. Note further that if the system $\Sigma(A, B)$ is $g$-optimizable for some $g \in \mathbb{R}$, then the system is $g^{\prime}$-optimizable for every $g^{\prime} \geq g$.

Definition 3.2. Let $F: D(F) \mapsto \mathbb{C}^{m}$. We call $F$ an LQ-stabilizing feedback at $g$ for the system $\Sigma(A, B)$ if there exists a $C_{0}$-semigroup $T_{F}(t)$ on $H$ with generator $A_{F}: D\left(A_{F}\right) \rightarrow H$ such that $F \in \mathcal{L}\left(D\left(A_{F}\right), \mathbb{C}^{m}\right), F$ is an admissible observation operator for $T_{F}(t)$,

$$
T_{F}(t) x_{0}=T(t) x_{0}+\int_{0}^{t} T(t-\rho) B F T_{F}(\rho) x_{0} d \rho
$$

for every $t \geq 0$ and any $x_{0} \in H$, and $\left\|T_{F}(t)\right\| \leq M e^{(g-\delta) t}$ for all $t \geq 0$ and some $M, \delta>0$. We call the feedback $F$ LQ-stabilizing, if it is LQ-stabilizing at zero.

Since $F$ is assumed to be an admissible observation operator for $T_{F}(t)$, we have that $F T_{F}(\cdot) x \in \boldsymbol{L}_{2}^{\text {loc }}\left(0, \infty ; \mathbb{C}^{m}\right)$ for all $x \in H$. Thus the integral in (7) is well-defined.

If an operator $F$ is stabilizing in the sense given in Rebarber [26] and Weiss and Curtain [37], then it is easy to see that it is also an LQ-stabilizing feedback. The 
difference between their definition and our Definition 3.2 is that we do not assume that $F$ is an admissible observation operator for $T(t)$. Therefore, in general we do not have the second perturbation equation

$$
T_{F}(t) x=T(t) x+\int_{0}^{t} T_{F}(t-\rho) B F T(\rho) x d \rho, \quad t \geq 0, x \in H,
$$

which holds if $F$ is stabilizing in their sense; see Weiss [36]. At the present it is unknown whether LQ-stabilizing feedbacks and stabilizing feedbacks in the sense of Rebarber are equivalent.

The following theorem shows that in our situation the system $\Sigma(A, B)$ is $g$ optimizable if and only if there exists a feedback which is LQ-stabilizing at $g$. So, we have equivalence between the boundedness of the LQ-cost functional (5) and our notion of stability. This inspired us to call our notion "LQ-stabilizing."

THEOREM 3.3. The following statements are equivalent.

(1) The system $\Sigma(A, B)$ is g-optimizable.

(2) There exists a feedback which is LQ-stabilizing at $g$ for the system $\Sigma(A, B)$.

Proof. By choosing $u(t)=F T_{F}(t) x_{0}$ it is easy to see that part (2) implies part (1). Thus it remains to prove that part (1) implies part (2). For $g$ equals zero, this implication is proved in Flandoli, Lasiecka, and Triggiani [8, Corollary 4.3 and Theorem 4.4]; see also Zwart [41]. For general $g \in \mathbb{R}$, it is easy to see that the cost $J^{g}\left(x_{0}, u\right)$ for the system $\Sigma(A, B)$ equals the cost $J\left(x_{0}, e^{-g} u\right)$ for the system $\Sigma(A-g I, B)$. Hence, the system $\Sigma(A, B)$ is $g$-optimizable if and only if the system $\Sigma(A-g I, B)$ is optimizable. For the system $\Sigma(A-g I, B)$ we use the result that we already have, and thus there exist an exponentially stable $C_{0}$-semigroup $T_{F}(t)$ and an admissible observation operator $F$ for $T_{F}(t)$ such that

$$
\begin{aligned}
T_{F}(t) x_{0} & =e^{-g t} T(t) x_{0}+\int_{0}^{t} e^{-g(t-\rho)} T(t-\rho) B F T_{F}(\rho) d \rho \\
& =e^{-g t}\left[T(t) x_{0}+\int_{0}^{t} T(t-\rho) B F e^{g \rho} T_{F}(\rho) d \rho\right] .
\end{aligned}
$$

This proves that $F$ is an LQ-stabilizing feedback at $g$ for the system $\Sigma(A, B)$.

For a $C_{0}$-semigroup $T(t)$ we define by $g_{b}(T)$ the growth bound of $T(t)$, i.e.,

$$
g_{b}(T):=\lim _{t \rightarrow \infty} \frac{1}{t} \log \|T(t)\| .
$$

If the system $\Sigma(A, B)$ is LQ-stabilizable, then we have the existence of an exponentially stable $C_{0}$-semigroup $T_{F}(t)$. The growth bound of this semigroup is denoted by $g_{b}^{F}$, i.e.,

$$
g_{b}^{F}:=g_{b}\left(T_{F}\right) .
$$

We call $g_{b}^{F}$ the closed-loop growth bound of the LQ-stabilizing feedback. If the system is optimizable, then $g_{b}^{F}$ depends on the LQ-stabilizing feedback $F$. However, $g_{b}^{F}$ will always be negative, and this is the essential property of the closed-loop growth bound that we will use in the following.

Proposition 3.4. Assume that $F$ is an LQ-stabilizing feedback for the system $\Sigma(A, B)$, and let $g_{b}^{F}$ be the corresponding closed-loop growth bound. Then we have the following. 
(1) For every $\varepsilon \in\left[0,-g_{b}^{F}\right)$ the feedback $F$ is LQ-stabilizing for the system $\Sigma(A+$ $\varepsilon I, B)$.

(2) For every $\varepsilon \in\left[0,-g_{b}^{F}\right)$ the feedback $F$ is $L Q$-stabilizing at $-\varepsilon$ for the system $\Sigma(A, B)$.

Proof. Let $\varepsilon \in\left[0,-g_{b}^{F}\right)$. By Remark $2.3 B$ is an admissible control operator for $e^{\varepsilon t} T(t)$. Let $F$ and $T^{F}(t)$ be as in Definition 3.2. Then Remark 2.3 implies that $F$ is an admissible observation operator for $e^{\varepsilon t} T^{F}(t)$. By the definition of $g_{b}^{F}$, the $C_{0}$-semigroup $T_{\varepsilon}^{F}(t):=e^{\varepsilon t} T^{F}(t)$ is exponentially stable.

1. From (7) we have that

$$
\begin{aligned}
T_{\varepsilon}^{F}(t) x_{0} & =e^{\varepsilon t} T_{F}(t) x_{0} \\
& =e^{\varepsilon t} T(t) x_{0}+\int_{0}^{t} e^{\varepsilon(t-\rho)} T(t-\rho) B F e^{\varepsilon \rho} T_{F}(\rho) d \rho \\
& =e^{\varepsilon t} T(t) x_{0}+\int_{0}^{t} e^{\varepsilon(t-\rho)} T(t-\rho) B F T_{\varepsilon}^{F}(\rho) d \rho .
\end{aligned}
$$

Since $A+\varepsilon I$ is the infinitesimal generator of the $C_{0}$-semigroup $e^{\varepsilon t} T(t)$, we see that $F$ is LQ-stabilizing for the system $\Sigma(A+\varepsilon I, B)$;

2. For all $\gamma>0$, there exists a $M_{\gamma}>0$ such that

$$
\left\|T_{F}(t)\right\| \leq M_{\gamma} e^{\left(g_{b}^{F}+\gamma\right) t} .
$$

We choose $\gamma>0$ such that $g_{b}^{F}+\gamma<-\varepsilon$ holds, and we define $\delta:=-\varepsilon-g_{b}^{F}-\gamma$. Then $\delta>0$ and $g_{b}^{F}+\gamma=-\varepsilon-\delta$, and hence by Definition 3.2 the feedback $F$ is LQ-stabilizing at $-\varepsilon$ for the system $\Sigma(A, B)$.

Next, we show that the spectral subset $\sigma_{g_{b}^{F}}^{+}(A)$ is of a special form if $F$ is a LQ-stabilizing feedback.

Proposition 3.5. Assume that $F$ is an LQ-stabilizing feedback for the system $\Sigma(A, B)$, and let $g_{b}^{F}$ be the closed-loop growth bound. Then the spectral subset $\sigma_{g_{b}^{F}}^{+}(A)$ consists only of point spectrum with finite algebraic multiplicity, and the possible finite accumulation points of the set $\sigma_{g_{b}^{F}}^{+}(A)$ must lie on the line with real part equal to $g_{b}^{F}$.

Proof. Let $\tau \in\left(g_{b}^{F}, 0\right)$ and $\varepsilon \in\left(-\tau,-g_{b}^{F}\right)$. Then Proposition 3.4 and Theorem 3.3 imply that system $\Sigma(A, B)$ is $\varepsilon$-optimizable. Now Rebarber and Zwart [28, Theorem 2.11 imply that the spectral subset $\sigma_{\tau}^{+}(A)$ consists only of point spectrum with finite algebraic multiplicity and contains no finite accumulation point. Since $\tau \in\left(g_{b}^{F}, 0\right)$ is arbitrary, this implies the statement.

Note that it is possible that the spectrum of $A$ possesses an accumulation point $\lambda_{0}$ with $\operatorname{Re}\left(\lambda_{0}\right)=g_{b}^{F}$.

In the following proposition we rewrite LQ-stabilizability in an equivalent way in the frequency domain. We show that LQ-stabilizability implies a useful representation of $x_{0} \in H$, the so-called $(\xi, \omega)$-representation. This was introduced for finitedimensional systems by Hautus [12] and for infinite-dimensional systems by Zwart [40].

Proposition 3.6.

1. If for every $x_{0} \in H$ there exist $\xi \in \boldsymbol{H}_{2}\left(\mathbb{C}_{0}^{+} ; H\right)$ and $\omega \in \boldsymbol{H}_{2}\left(\mathbb{C}_{0}^{+} ; \mathbb{C}^{m}\right)$ such that

$$
x_{0}=(s I-A) \xi(s)-B \omega(s), \quad s \in \mathbb{C}_{0}^{+},
$$


then the system $\Sigma(A, B)$ is optimizable, and hence there exists an LQ-stabilizing feedback.

2. Assume that there exists an LQ-stabilizing feedback for the system $\Sigma(A, B)$, and let $g_{b}^{F}$ be its closed-loop growth bound. Then for every $\sigma \in\left(g_{b}^{F}, 0\right)$ there exists a constant $C>0$ such that for every $x_{0} \in H$ there exist $\xi \in$ $\boldsymbol{H}_{\infty}\left(\mathbb{C}_{\sigma}^{+} ; H\right) \cap \boldsymbol{H}_{2}\left(\mathbb{C}_{\sigma}^{+} ; H\right)$ and $\omega \in \boldsymbol{H}_{\infty}\left(\mathbb{C}_{\sigma}^{+} ; \mathbb{C}^{m}\right) \cap \boldsymbol{H}_{2}\left(\mathbb{C}_{\sigma}^{+} ; \mathbb{C}^{m}\right)$ with

$$
x_{0}=(s I-A) \xi(s)-B \omega(s), \quad s \in \mathbb{C}_{\sigma}^{+},
$$

and

$$
\max \left\{\|\xi\|_{\boldsymbol{H}_{\infty}\left(\mathbb{C}_{\sigma}^{+} ; H\right)},\|\xi\|_{\boldsymbol{H}_{2}\left(\mathbb{C}_{\sigma}^{+} ; H\right)},\|\omega\|_{\boldsymbol{H}_{\infty}\left(\mathbb{C}_{\sigma}^{+} ; \mathbb{C}^{m}\right)},\|\omega\|_{\boldsymbol{H}_{2}\left(\mathbb{C}_{\sigma}^{+} ; \mathbb{C}^{m}\right)}\right\} \leq C\left\|x_{0}\right\|_{H} .
$$

Proof. By [33, Remark 3.12] it follows that for every $u \in \boldsymbol{L}_{2}\left(0, \infty ; \mathbb{C}^{m}\right)$ the function $\Phi_{u}(t):=\int_{0}^{t} T(t-\rho) B u(\rho) d \rho$ is Laplace-transformable with Laplace transform

$$
\hat{\Phi}_{u}(s)=(s I-A)^{-1} B \omega(s), \quad \operatorname{Re}(s) \geq r \text { for some } r \in \mathbb{R},
$$

where $\omega$ is the Laplace transform of $u$.

1. Let $x_{0} \in H$ be arbitrary. Since $\xi \in \boldsymbol{H}_{2}\left(\mathbb{C}_{0}^{+} ; H\right)$ and $\omega \in \boldsymbol{H}_{2}\left(\mathbb{C}_{0}^{+} ; \mathbb{C}^{m}\right)$, by the Paley-Wiener theorem (see, e.g., [3, Theorem A.6.21]) there exist unique $u \in \boldsymbol{L}_{2}\left(0, \infty ; \mathbb{C}^{m}\right)$ and $x \in \boldsymbol{L}_{2}(0, \infty ; H)$ such that $\omega$ is the Laplace transform of $u$ and $\xi$ is the Laplace transform of $x$. Applying Laplace transforms to the function

$$
T(t) x_{0}+\int_{0}^{t} T(t-\rho) B u(\rho) d \rho, \quad t \geq 0,
$$

we get

$$
(s I-A)^{-1} x_{0}+(s I-A)^{-1} B \omega(s), \quad \operatorname{Re}(s) \geq r \text { for some } r \in \mathbb{R} .
$$

Since $\xi(s)=(s I-A)^{-1} x_{0}+(s I-A)^{-1} B \omega(s), \operatorname{Re}(s)>\max \{r, 0\}$, the uniqueness of the Laplace transform implies

$$
x(t)=T(t) x_{0}+\int_{0}^{t} T(t-\rho) B u(\rho) d \rho .
$$

This proves that $x$ is the state trajectory corresponding to $x_{0}$ and $u$ and thus the system $\Sigma(A, B)$ is optimizable. By Theorem 3.3 we have the existence of an LQ-stabilizing feedback.

2. Let $F$ be a LQ-stabilizing feedback from Definition 3.2. Taking the Laplace transform of (7), we get

$$
\left(s I-A_{F}\right)^{-1} x_{0}=(s I-A)^{-1} x_{0}+(s I-A)^{-1} B F\left(s I-A_{F}\right)^{-1} x_{0} .
$$

Since $T_{F}(t)$ has growth bound $g_{b}^{F}$, it is easy to see that

$$
\left\|\left(s I-A_{F}\right)^{-1} x_{0}\right\|_{\boldsymbol{H}_{\infty}\left(\mathbb{C}_{\sigma}^{+} ; H\right)} \leq C_{1}\left\|x_{0}\right\|_{H},
$$

and by using the Paley-Wiener theorem [3, Theorem A.6.21] we get

$$
\left\|\left(s I-A_{F}\right)^{-1} x_{0}\right\|_{\boldsymbol{H}_{2}\left(\mathbb{C}_{\sigma}^{+} ; H\right)} \leq C_{2}\left\|x_{0}\right\|_{H} .
$$

Since $F$ is an admissible observation operator for $T_{F}(t)$, similar results hold for $F\left(s I-A_{F}\right)^{-1} x_{0}$. Define $\xi(s):=\left(s I-A_{F}\right)^{-1} x_{0}$ and $\omega(s):=F\left(s I-A_{F}\right)^{-1} x_{0}$. Multiplying (11) with $(s I-A)$ and using the definition of $\xi$ and $\omega$, we obtain (10). 
We end this section with the definition and some simple properties of exact controllability in finite time.

Definition 3.7. The system $\Sigma(A, B)$ is called exactly controllable in finite time if there exists a time $t_{0} \in(0, \infty)$ such that for every $x \in H$ we can find an input $u \in \boldsymbol{L}_{2}\left(0, t_{0} ; \mathbb{C}^{m}\right)$ such that

$$
x=\int_{0}^{t_{0}} T\left(t_{0}-\rho\right) B u(\rho) d \rho .
$$

Lemma 3.8. Assume that the system $\Sigma(A, B)$ is exactly controllable in finite time. Then for every $\tau \in \mathbb{R}$ the system $\Sigma(A+\tau I, B)$ also is exactly controllable in finite time.

The following proposition shows that exact controllability in finite time implies $g$-optimizability for every $g \in \mathbb{R}$.

Proposition 3.9. If the system $\Sigma(A, B)$ is exactly controllable in finite time, then it also is g-optimizable for every $g \in \mathbb{R}$.

The proof of Lemma 3.8 and Proposition 3.9 is quite easy and left to the reader. We conclude this section by proving that the spectrum of an exactly controllable system is of a very special form.

THEOREM 3.10. If the system $\Sigma(A, B)$ is exactly controllable in finite time, then the spectrum of $A$ is pure point spectrum and it contains no (finite) accumulation point.

Proof. Let $\gamma \in \mathbb{R}$ be arbitrary. Then by Lemma 3.8 we see that the system $\Sigma(A+\gamma I, B)$ is exactly controllable in finite time as well. Proposition 3.9 implies that $\Sigma(A+\gamma I, B)$ is optimizable. Now from Theorem 3.3 and Proposition 3.5 we obtain that $\sigma_{0}^{+}(A+\gamma I)$ consists only of point spectrum with no finite accumulation point. Since $\sigma_{-\gamma}^{+}(A)=\sigma_{0}^{+}(A+\gamma I)$, and since $\gamma$ is arbitrary, the assertion follows.

4. Decompositions of $\boldsymbol{C}_{\mathbf{0}}$-semigroups. For the formulation of Theorem 5.3 we need to write our system into two subsystems. Properties of such a decomposition are given next.

Definition 4.1. We call a closed subspace $V$ of $H T(t)$-invariant, if $T(t) V \subset V$, for all $t \geq 0$.

In Kurtz [19] it is shown that a closed subspace $V$ is $T(t)$-invariant if and only if $(\lambda I-A)^{-1} V \subset V$ for all $\lambda \in \rho_{\infty}(A)$; see also Curtain and Zwart [3, Lemma 2.5.6].

Lemma 4.2. Let $V$ be a $T(t)$-invariant subspace of $H$ and let $P_{V} \in \mathcal{L}(H)$ be a projection from $H$ onto $V$. Define $W:=N\left(P_{V}\right)$ and $P_{W}:=I-P_{V}$. Then the following hold:

1. The operator

$$
T_{V}(t) x:=T(t) x, \quad t \geq 0, x \in V,
$$

defines a $C_{0}$-semigroup on $V$ with its generator $A_{V}$ given by

$$
A_{V} x:=A x, \quad x \in D\left(A_{V}\right):=D(A) \cap V .
$$

Furthermore, $\rho_{\infty}(A) \subset \rho\left(A_{V}\right)$, and for every $v \in V$ and $s \in \rho_{\infty}(A)$ we have $\left(s I-A_{V}\right)^{-1} v=(s I-A)^{-1} v$.

2. Let $V_{-1}$ be the completion of $V$ with respect to the norm $\left\|\left(\beta I-A_{V}\right)^{-1} \cdot\right\|_{V}$. Then $V_{-1} \subset H_{-1}$ and $\|v\|_{V_{-1}}=\|v\|_{H_{-1}}$ for every $v \in V_{-1}$. 
3. The operator

$$
T_{W}(t) x:=P_{W} T(t) x, \quad t \geq 0, x \in W,
$$

defines a $C_{0}$-semigroup on $W$.

Proof.

1. Follows immediately from Pazy [22, page 123] and [19].

2. From the first part it follows that $\left\|\left(s I-A_{V}\right)^{-1} v\right\|=\left\|(s I-A)^{-1} v\right\|$ for every $v \in V$. Now the definition of a completion of a normed vector space shows that $V_{-1} \subset H_{-1}$ and $\|v\|_{V_{-1}}=\|v\|_{H_{-1}}$ for every $v \in V_{-1}$.

3. It is easy to see that $T_{W}(0)=I_{W}$ holds. Since $V$ is a $T(t)$-invariant subspace, we have $P_{W} T(t) P_{V}=0$. Thus, for $t, s \geq 0$ and $x \in W$ we obtain

$$
\begin{aligned}
T_{W}(t) T_{W}(s) x & =P_{W} T(t) P_{W} T(s) x=P_{W} T(t) T(s) x-P_{W} T(t)\left(I-P_{W}\right) T(s) x \\
& =P_{W} T(t+s) x-P_{W} T(t) P_{V} T(s) x=T_{W}(s+t) x .
\end{aligned}
$$

Since the strong continuity of $T(t)$ immediately implies that $T_{W}(t)$ is strongly continuous, $T_{W}(t)$ is a $C_{0}$-semigroup on $W$.

Lemma 4.3. Let $V$ be a $T(t)$-invariant subspace of $H$, and let $P_{V} \in \mathcal{L}(H)$ be a projection from $H$ onto $V$. Define $W:=N\left(P_{V}\right)$ and $P_{W}:=I-P_{V}$. Then there exists a unique admissible control operator $B_{W} \in \mathcal{L}\left(\mathbb{C}^{m}, W_{-1}\right)$ for $T_{W}(t)$ on $W$, where $T_{W}(t) x:=P_{W} T(t) x$ for $t \geq 0$ and $x \in W$, with the property that

$$
\int_{0}^{t} T_{W}(t-\rho) B_{W} u(\rho) d \rho=P_{W} \int_{0}^{t} T(t-\rho) B u(\rho) d \rho
$$

for every $t \geq 0$ and $u \in \boldsymbol{L}_{2}\left(0, t ; \mathbb{C}^{m}\right)$. This unique operator $B_{W}$ is given by

$$
B_{W} u:=\lim _{\tau \rightarrow 0} \frac{1}{\tau} P_{W} \int_{0}^{\tau} T(\tau-\rho) B u d \rho, \quad u \in \mathbb{C}^{m},
$$

where the limit is taken in $W_{-1}$.

Additionally, if $\Sigma(A, B)$ is exactly controllable in finite time, then $\Sigma\left(A_{W}, B_{W}\right)$ also is exactly controllable in finite time. Here $A_{W}$ is the generator of the $C_{0}$ semigroup $T_{W}(t)$.

Proof. Let $\mathcal{B}_{t} \in \mathcal{L}\left(\boldsymbol{L}_{2}\left(0, \infty ; \mathbb{C}^{m}\right), H\right)$ be the operator as introduced in Definition 2.1. For $u, v \in L_{2}\left(0, \infty ; \mathbb{C}^{m}\right)$ we define $u \stackrel{\tau}{\diamond} v$ as

$$
(u \diamond \underset{\tau}{\diamond})(t)= \begin{cases}u(t), & t \in[0, \tau] \\ v(t-\tau), & t>\tau .\end{cases}
$$

The admissibility of $B$ for the $C_{0}$-semigroup $T(t)$ implies (see [33]) that

$$
\mathcal{B}_{\tau+t}(u \diamond v)=T(t) \mathcal{B}_{\tau} u+\mathcal{B}_{t} v
$$

Define $\mathcal{B}_{t}^{W} \in \mathcal{L}\left(\boldsymbol{L}_{2}\left(0, \infty ; \mathbb{C}^{m}\right), W\right), t \geq 0$, by

$$
\mathcal{B}_{t}^{W}:=P_{W} \mathcal{B}_{t} .
$$

Since $V$ is a $T(t)$-invariant subspace of $H$, we have that $P_{W} T(t) P_{V}=0, t \geq 0$. Thus for every $\tau, t \geq 0$ and every $u, v \in \boldsymbol{L}_{2}\left(0, \infty ; \mathbb{C}^{m}\right)$ we obtain

$$
\begin{aligned}
\mathcal{B}_{\tau+t}^{W}(u \diamond v) & =P_{W} \mathcal{B}_{\tau+t}(u \stackrel{\diamond}{\diamond} v)=P_{W} T(t) \mathcal{B}_{\tau} u+P_{W} \mathcal{B}_{t} v=P_{W} T(t) \mathcal{B}_{\tau} u+\mathcal{B}_{t}^{W} v \\
& =P_{W} T(t) P_{W} \mathcal{B}_{\tau} u+P_{W} T(t) P_{V} \mathcal{B}_{\tau} u+\mathcal{B}_{t}^{W} v=T_{W}(t) \mathcal{B}_{\tau}^{W} u+\mathcal{B}_{t}^{W} v .
\end{aligned}
$$


Now [33, Theorem 3.9] implies that there exists a unique $B_{W} \in \mathcal{L}\left(\mathbb{C}^{m}, W_{-1}\right)$ such that

$$
\mathcal{B}_{t}^{W} u=\int_{0}^{t} T_{W}(t-\rho) B_{W} u(\rho) d \rho,
$$

for $t \geq 0$ and $u \in \boldsymbol{L}_{2}\left(0, \infty ; \mathbb{C}^{m}\right)$, and this unique operator $B_{W}$ is given by (13). Thus $B_{W}$ is an admissible control operator for $T_{W}(t)$ and (12) holds.

If $\Sigma(A, B)$ is exactly controllable in finite time, then there exists a time $t_{0}>0$ such that for every $x \in W$ there is an input $u \in \boldsymbol{L}_{2}\left(0, t_{0} ; \mathbb{C}^{m}\right)$ such that

$$
x=\int_{0}^{t_{0}} T\left(t_{0}-\rho\right) B u(\rho) d \rho .
$$

From (12) it follows directly that the system $\Sigma\left(A_{W}, B_{W}\right)$ is exactly controllable in finite time as well.

If $P$ is a projection that commutes with the $C_{0}$-semigroup $T(t)$, we have $H=$ $N(P) \oplus \operatorname{Im}(P)$ and both $N(P)$ and $\operatorname{Im}(P)$ are closed $T(t)$-invariant subspaces. In the following we denote by $T_{u}(t)$ and $T_{s}(t)$ the restricted $C_{0}$-semigroups on $H_{u}:=N(P)$ and $H_{s}:=\operatorname{Im}(P)$, respectively. Moreover, the generators of the $C_{0}$-semigroups $T_{u}(t)$ and $T_{s}(t)$ are denoted by $A_{u}$ and $A_{s}$. By $\left[H_{s}\right]_{-1}\left(\left[H_{u}\right]_{-1}\right)$ we denote the completion of $H_{s}\left(H_{u}\right)$ with respect to the norm $\left\|\left(\beta I-A_{s}\right)^{-1} \cdot\right\|_{H_{s}}\left(\left\|\left(\beta I-A_{u}\right)^{-1} \cdot\right\|_{H_{u}}\right)$.

The existence of such a projection $P$ is very helpful since it reduces the semigroup into two (possibly simpler) semigroups $T_{s}(t)$ and $T_{u}(t)$.

LemMA 4.4. Let $T(t)$ be a $C_{0}$-semigroup on $H$ and $B \in \mathcal{L}\left(\mathbb{C}^{m}, H_{-1}\right)$ an admissible control operator for $T(t)$. Moreover, let $P \in \mathcal{L}(H)$ be a projection that commutes with $T(t), t \geq 0$. Then we have the following.

1. $P$ has a unique continuous extension $\tilde{P}$ in $\mathcal{L}\left(H_{-1}\right)$ with $\operatorname{Im}(\tilde{P})=\left[H_{s}\right]_{-1}$ and $N(\tilde{P})=\left[H_{u}\right]_{-1}$. Moreover, $\tilde{P}$ is a projection and $\tilde{P}$ commutes with $T(t)$ and $A$.

2. $\tilde{P} B \in \mathcal{L}\left(\mathbb{C}^{m},\left[H_{s}\right]_{-1}\right)$ is an admissible control operator for $T_{s}(t)$ on $H_{s}$ with the property

$$
\int_{0}^{t} T_{s}(t-\rho) \tilde{P} B u(\rho) d \rho=P \int_{0}^{t} T(t-\rho) B u(\rho) d \rho,
$$

for $t \geq 0$ and $u \in \boldsymbol{L}_{2}\left(0, t ; \mathbb{C}^{m}\right)$.

3. $(I-\tilde{\tilde{P}}) B \in \mathcal{L}\left(\mathbb{C}^{m},\left[H_{u}\right]_{-1}\right)$ is an admissible control operator for $T_{u}(t)$ on $H_{u}$ with the property

$$
\int_{0}^{t} T_{u}(t-\rho)(I-\tilde{P}) B u(\rho) d \rho=(I-P) \int_{0}^{t} T(t-\rho) B u(\rho) d \rho,
$$

for $t \geq 0$ and $u \in \boldsymbol{L}_{2}\left(0, t ; \mathbb{C}^{m}\right)$.

Proof.

1. Choose $\beta \in \rho(A)$ with $\operatorname{Re} \beta>g_{b}(T)$, where $g_{b}(T)$ is the growth bound of $T(t)$. Then we have

$$
(\beta I-A)^{-1} x=\int_{0}^{\infty} e^{-\beta t} T(t) x d t .
$$

Thus $P$ commutes with $(\beta I-A)^{-1}$, and hence also with $A$. Now [33, Proposition 3.3] shows that $P$ has a unique continuous extension $\tilde{P}$ in $\mathcal{L}\left(H_{-1}\right)$ and 
that $\tilde{P}$ commutes with $T(t)$ and $A$. It is now easy to see that $\tilde{P}$ again is a projection.

In order to prove $\operatorname{Im}(\tilde{P})=\left[H_{s}\right]_{-1}$, we first choose $x \in\left[H_{s}\right]_{-1}$. Then there exists a sequence $\left\{x_{n}\right\}_{n} \subset H_{s}$ with $x_{n} \rightarrow x$, as $n \rightarrow \infty$, in $\left[H_{s}\right]_{-1}$. Since $\tilde{P}$ is continuous, we get $\tilde{P} x_{n} \rightarrow \tilde{P} x$. On the other hand, $\tilde{P} x_{n}=P x_{n}=x_{n} \rightarrow x$, and thus $x \in \operatorname{Im}(\tilde{P})$. Conversely, we choose $x \in \operatorname{Im}(\tilde{P})$. Then there exists an element $z \in H_{-1}$ with $\tilde{P} z=x$ and a sequence $\left\{z_{n}\right\}_{n} \subset H_{\tilde{P}}$ with $z_{n} \rightarrow z$, as $n \rightarrow \infty$, in $H_{-1}$. Since $P z_{n} \in H_{s}$ and $P z_{n}=\tilde{P} z_{n} \rightarrow \tilde{P} z=x$, we get $x \in\left[H_{s}\right]_{-1}$. This proves $\operatorname{Im}(\tilde{P})=\left[H_{s}\right]_{-1}$. Finally, $N(\tilde{P})=\left[H_{u}\right]_{-1}$ can be proved in a similar manner.

2. By Lemma 4.3 there is a unique admissible control operator $B_{s} \in \mathcal{L}\left(\mathbb{C}^{m},\left[H_{s}\right]_{-1}\right)$ for $T_{s}(t)$ with the property that

$$
\int_{0}^{t} T_{s}(t-\rho) B_{s} u(\rho) d \rho=P \int_{0}^{t} T(t-\rho) B u(\rho) d \rho,
$$

for every $t \geq 0$ and $u \in \boldsymbol{L}_{2}\left(0, t ; \mathbb{C}^{m}\right)$. Using [33], we see that $B$ is given by

$$
B u=\lim _{\tau \rightarrow \infty} \frac{1}{\tau} \int_{0}^{t} T(t-\rho) B u d \rho, \quad u \in \mathbb{C}^{m},
$$

where the limit is taken in $H_{-1}$. Since $\tilde{P} \in \mathcal{L}\left(H_{-1}\right)$, we get

$$
\tilde{P} B u=\lim _{\tau \rightarrow \infty} \frac{1}{\tau} P \int_{0}^{t} T(t-\rho) B u d \rho, \quad u \in \mathbb{C}^{m} .
$$

By Lemma $4.2,\left[H_{s}\right]_{-1} \subset H_{-1}$ and the norms coincide. Now by Lemma 4.3 we get $B_{s}=\tilde{P} B$, which completes the proof.

3. The proof for $T_{u}(t)$ is similar to the proof of part 2 .

5. Equivalent conditions for optimizability. In this section, we develop equivalent conditions for optimizability. We will see that under a weak assumption the system $\Sigma(A, B)$ is optimizable if and only if we can split it into a part that is exponentially stable and a part that is exactly controllable in finite time. This sufficient condition of optimizability was obtained fairly early in 1975 by Triggiani [31] for infinite-dimensional systems with a bounded control operator, under the extra assumption of finitely many unstable eigenvalues. We now show that this holds for admissible control operators for $T(t)$.

THEOREM 5.1. Assume that there exists a projection $P \in \mathcal{L}(H)$ such that

(1) $P$ commutes with the semigroup $T(t)$;

(2) $T_{s}(t):=P T(t)$ is an exponentially stable $C_{0}$-semigroup on $H_{s}:=\operatorname{Im}(P)$;

(3) $\Sigma\left(A_{u},(I-\tilde{P}) B\right)$ is exactly controllable in finite time. Here $A_{u}$ is the generator of the $C_{0}$-semigroup $T_{u}(t):=(I-P) T(t)$ on $H_{u}:=N(P)$.

Then the system $\Sigma(A, B)$ is optimizable.

Proof. Let $x_{0} \in H$ be arbitrary. Since $\Sigma\left(A_{u},(I-\tilde{P}) B\right)$ is exactly controllable in finite time there exist a number $t_{0}>0$ and an input $\tilde{u} \in \boldsymbol{L}_{2}\left(0, t_{0} ; \mathbb{C}^{m}\right)$ such that

$$
-T_{u}\left(t_{0}\right)(I-P) x_{0}=\int_{0}^{t_{0}} T_{u}\left(t_{0}-\rho\right)(I-\tilde{P}) B \tilde{u}(\rho) d \rho .
$$

Define $u \in \boldsymbol{L}_{2}\left(0, \infty ; \mathbb{C}^{m}\right)$ by

$$
u(s):= \begin{cases}\tilde{u}(s), & s \leq t_{0} \\ 0, & s>t_{0}\end{cases}
$$


Then for $t \geq t_{0}$ the state trajectory corresponding to the initial state $x_{0}$ and the input function $u$ satisfies

$$
\begin{aligned}
& x(t)=T(t) x_{0}+\int_{0}^{t} T(t-\rho) B u(\rho) d \rho
\end{aligned}
$$

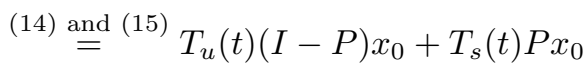

$$
\begin{aligned}
& +\int_{0}^{t} T_{u}(t-\rho)(I-\tilde{P}) B u(\rho) d \rho+\int_{0}^{t} T_{s}(t-\rho) \tilde{P} B u(\rho) d \rho \\
& =T_{u}\left(t-t_{0}\right) T_{u}\left(t_{0}\right)(I-P) x_{0}+T_{s}(t) P x_{0} \\
& +T_{u}\left(t-t_{0}\right) \int_{0}^{t_{0}} T_{u}\left(t_{0}-\rho\right)(I-\tilde{P}) B \tilde{u}(\rho) d \rho \\
& +T_{s}\left(t-t_{0}\right) \int_{0}^{t_{0}} T_{s}\left(t_{0}-\rho\right) \tilde{P} B \tilde{u}(\rho) d \rho \\
& \stackrel{(17)}{=} \quad T_{s}(t) P x_{0}+T_{s}\left(t-t_{0}\right) \int_{0}^{t_{0}} T_{s}\left(t_{0}-\rho\right) \tilde{P} B \tilde{u}(\rho) d \rho .
\end{aligned}
$$

Now the exponential stability of $T_{s}(t)$ implies $x \in \boldsymbol{L}_{2}(0, \infty ; H)$, and thus the system $\Sigma(A, B)$ is optimizable.

To show that the converse of Theorem 5.1 holds, we need an extra assumption.

DeFInITION 5.2. We say that the operator A satisfies the spectrum decomposition assumption at $g(\operatorname{SDA}(g))$, if there exist numbers $g_{1}<g<g_{2}$ such that

$$
\mathbb{C}_{g_{1}}^{+} \cap \mathbb{C}_{g_{2}}^{-} \subset \rho(A) .
$$

We say that $A$ satisfies the $S D A$, if it satisfies $S D A(0)$.

If an operator $A$ satisfies the $\operatorname{SDA}(g)$ in the sense given in Curtain and Zwart [3], then $A$ satisfies the SDA at $g$ in the sense of Definition 5.2. The difference between their definition and Definition 5.2 is that we do not assume that $\sigma_{g}^{+}(A)$ is compact.

The result that we want to prove in this section is the following.

THEOREM 5.3. Assume that the system $\Sigma(A, B)$ is optimizable and that $A$ satisfies SDA. Then there exists a projection $P$ such that

(1) $P$ commutes with the semigroup $T(t)$,

(2) $T_{s}(t):=P T(t)$ is an exponentially stable $C_{0}$-semigroup on $H_{s}:=\operatorname{Im}(P)$,

(3) $T_{u}(t):=(I-P) T(t)$ is a $C_{0}$-group on $H_{u}:=N(P)$,

(4) $-A_{u}$ generates an exponentially stable $C_{0}$-semigroup,

(5) $\Sigma\left(A_{u},(I-\tilde{P}) B\right)$ is exactly controllable in finite time.

REMARK 5.4. There are examples of optimizable systems $\Sigma(A, B)$ for which $A$ does not satisfy the SDA, but for every $g<0$ there exists an $\varepsilon \in(0,-g)$ such that A satisfies the SDA at $-\varepsilon$. If this is the case, then using Theorem 3.3, Proposition 3.4, and Theorem 5.3, we get a result similar to that in Theorem 5.3; namely, there exists a projection $P$ such that 1-3 and 5 of Theorem 5.3 are satisfied and additionally $-A_{u}-\varepsilon I$ generates an exponentially stable $C_{0}$-semigroup.

A projection $P \in \mathcal{L}(H)$ satisfying properties (1)-(4) of Theorem 5.3 is also known as dichotomic projection; see [24]. The proof of Theorem 5.3 is given at the end of this section.

Desch and Schappacher [5], Jacobson and Nett [17] and Nefedov and Sholokhovich [20] have proved Theorem 5.3 for bounded control operators $B$, i.e., $B \in \mathcal{L}\left(\mathbb{C}^{m}, H\right)$. In this situation, for every (LQ)-stabilizing feedback $F$, the spectral subset $\sigma_{g_{b}^{F}}^{+}(A)$ 
consists only of finitely many points. Hence, the SDA is satisfied or at least $\mathrm{SDA}(g)$ is satisfied for $g$ arbitrarily close to zero. Example 5.11 shows that the assumptions made in Theorem 5.1 do not imply that $A$ satisfies the SDA at $g$ for some $g \leq 0$. In the following we will see that the assumption that $A$ satisfies the SDA is not very restrictive, because there are a lot of situations where this assumption holds automatically; see Proposition 5.9.

Definition 5.5. We call the $C_{0}$-semigroup $T(t)$ completely unstable if

$$
\left\{x \in H: T(\cdot) x \in \boldsymbol{L}_{2}(0, \infty ; H)\right\}=\{0\} .
$$

For example, if $A$ generates a $C_{0}$-group $T(t)$ and $-A$ generates a bounded $C_{0^{-}}$ semigroup, then $T(t)$ is completely unstable.

As a corollary of Theorem 5.3 we show that under the assumption that $A$ generates a completely unstable $C_{0}$-semigroup, the system $\Sigma(A, B)$ is optimizable if and only if the system $\Sigma(A, B)$ is exactly controllable in finite time.

Corollary 5.6. If $A$ generates a completely unstable $C_{0}$-semigroup, then the following statements are equivalent.

(1) The system $\Sigma(A, B)$ is optimizable.

(2) There exists an $L Q$-stabilizing feedback for the system $\Sigma(A, B)$.

(3) The system $\Sigma(A, B)$ is exactly controllable in finite time.

Moreover, if one of these statements holds, then $T(t)$ is a $C_{0}$-group on $H$, and $A$ satisfies the SDA at any point between the closed-loop growth bound and zero.

Proof. The equivalence between part 1 and part 2 is proved in Theorem 3.3. Proposition 3.9 shows that part 3 implies part 1 . We now prove that part 2 implies part 3. Let $F$ be an LQ-stabilizing feedback for the system $\Sigma(A, B)$. Proposition 3.5 implies that the spectral subset $\sigma_{g_{b}^{F}}^{+}(A)$ consists only of point spectrum. Let us assume that there is a $\lambda \in \mathbb{C}_{g_{b}^{F}}^{+} \cap \mathbb{C}_{0}^{-} \cap \sigma(A)$ and $x_{\lambda}$ a corresponding eigenvector. In $[22$, p. 46] it is shown that $e^{\lambda t}$ is an eigenvalue of $T(t)$ with eigenvector $x_{\lambda}$. This implies that $T(\cdot) x_{\lambda}=e^{\lambda t} x_{\lambda} \in \boldsymbol{L}_{2}(0, \infty ; H)$, which is in contradiction with the fact that $T(t)$ is completely unstable. Thus $\mathbb{C}_{g_{b}^{F}}^{+} \cap \mathbb{C}_{0}^{-} \subset \rho(A)$ and so $A$ satisfies the $\operatorname{SDA}(-\varepsilon)$ for any $\varepsilon \in\left(0,-g_{b}^{F}\right)$. Remark 5.4 now implies the existence of a projection $P$ such that $1-3$ and 5 of Theorem 5.3 are satisfied. Since $T_{s}(t)=P T(t)=T(t) P$, we get $T(\cdot) x \in L_{2}(0, \infty ; H)$ for every $x \in H_{s}$. Using the complete instability of our system this can only happen if $H_{s}=0$. Therefore, $H_{u}=H$ and $T(t)$ equals $T_{u}(t)$ which implies that $T(t)$ is a $C_{0}$-group and that the system $\Sigma(A, B)$ is exactly controllable in finite time.

The result presented in the previous corollary is closely related to Russell's principle on exact controllability. Russell [29] proved exact controllability for a system governed by the wave equation by showing that the system was stabilizable and backwards stabilizable. The definition of stabilizability used in [29] is stronger than the definition used in this paper. Rebarber and Weiss [27] extended Russell's result to our notion of stabilizability. Their result is closely related to the corollary above, but it is neither a consequence nor a generalization of our result.

EXAMPLE 5.7. Consider the right-shift semigroup $T(t)$ on $\boldsymbol{L}_{2}(0, \infty)$ with generator $A$. This semigroup satisfies $\left\|T(t) x_{0}\right\|=\left\|x_{0}\right\|$ for every $x_{0} \in \boldsymbol{L}_{2}(0, \infty)$. Hence the right shift semigroup on $\boldsymbol{L}_{2}(0, \infty)$ is completely unstable. Since this $C_{0}$-semigroup is not a $C_{0}$-group, Corollary 5.6 implies that there cannot exist an admissible finite-rank control operator $B$ for $T(t)$ such that the system $\Sigma(A, B)$ is optimizable. 
Definition 5.8. We call a $C_{0}$-semigroup $T(t)$ eventually norm continuous if there exists a constant $t^{\prime} \geq 0$ such that the function $t \rightarrow T(t)$ from $\left(t^{\prime}, \infty\right)$ into $\mathcal{L}(H)$ is operator-norm continuous.

Examples for eventually norm continuous $C_{0}$-semigroups are holomorphic, differentiable, norm continuous, and compact $C_{0}$-semigroups; see Arendt et al. [1, p. 41].

Proposition 5.9. Assume that $F$ is an LQ-stabilizing feedback for the system $\Sigma(A, B)$, and let $g_{b}^{F}$ be its closed-loop growth bound. If the $C_{0}$-semigroup $T(t)$ is eventually norm continuous, then for every $\tau \in\left(g_{b}^{F}, 0\right)$, the set $\sigma_{\tau}^{+}(A)$ contains only finitely many points, and thus A satisfies $S D A(g)$ for all but finitely many $g>g_{b}^{F}$.

Proof. By Proposition 3.5 the spectral subset $\sigma_{g_{b}^{F}}^{+}(A)$ contains no finite accumulation point. Let $\tau \in\left(g_{b}^{F}, 0\right)$. Since by [1, A-II Theorem 1.20] the set $\{\lambda \in$ $\sigma(A) \mid \operatorname{Re}(\lambda) \geq \tau\}$ is bounded, we have that $\sigma_{\tau}^{+}(A)$ contains only finitely many points. Thus the assertion follows.

In the special case where $A$ is a Riesz-spectral operator we are able to prove Theorem 5.3 without the assumption that $A$ satisfies the SDA. For the definition of a Riesz-spectral operator we refer the reader to [3, Definition 2.3.4].

THEOREM 5.10. Assume that $F$ is an LQ-stabilizing feedback for the system $\Sigma(A, B)$ and that $A$ is a Riesz-spectral operator. Let $g$ be negative and larger than the closed-loop growth bound. Then there exists a projection $P \in \mathcal{L}(H)$ such that $1-3$ and 5 of Theorem 5.3 are satisfied and additionally the growth bound of the $C_{0}$-semigroup generated by $-A_{u}$ is at most $-g$.

Proof. By the definition of a Riesz-spectral operator, there exist sequences $\left(\lambda_{n}\right)_{n} \subset \mathbb{C},\left(\phi_{n}\right)_{n} \subset H$, and $\left(\psi_{n}\right)_{n} \subset H$, with $\left\langle\phi_{n}, \psi_{m}\right\rangle=\delta_{n, m}$, such that

$$
\begin{aligned}
A z & :=\sum_{n \in \mathbb{N}} \lambda_{n}\left\langle z, \psi_{n}\right\rangle \phi_{n}, \quad z \in H, \\
D(A) & :=\left\{\left.z \in H\left|\sum_{n \in \mathbb{N}}\right| \lambda_{n}\right|^{2}\left|\left\langle z, \psi_{n}\right\rangle\right|^{2}<\infty\right\} .
\end{aligned}
$$

Let $T_{F}(t)$ and $F$ be the operators given by Definition 3.2. Defining $S:=\{n \in \mathbb{N} \mid$ $\left.\operatorname{Re}\left(\lambda_{n}\right) \leq g\right\}$, it is easy to see that

$$
P z:=\sum_{n \in S}\left\langle z, \psi_{n}\right\rangle \phi_{n}
$$

is a projection operator on $H$ which commutes with $A$ and $T(t), t \geq 0$. Moreover, by [3, Theorem 2.3.5] we get that

(1) $T_{s}(t):=P T(t)$ is an exponentially stable $C_{0}$-semigroup on $H_{s}:=\operatorname{Im}(P)$,

(2) $T_{u}(t):=(I-P) T(t)$ is a $C_{0}$-group on $H_{u}:=N(P)$,

(3) $\left\|T_{u}(t) x\right\| \leq M e^{g t}\|x\|$ for all $x \in H_{u}, t \leq 0$,

where $M>0$ is independent of $x$ and $t$. Lemma 4.4 now shows that $(I-\tilde{P}) B$ is an admissible control operator for $T_{u}(t)$. Thus it remains to prove that $\Sigma\left(A_{u},(I-\tilde{P}) B\right)$ is exactly controllable in finite time. Since $T_{-g}^{F}(t):=e^{-g t} T_{F}(t)$ is exponentially stable and $T_{u}(t)$ satisfies the inequality in outcome (3), the operator $\left.T_{u}(-t)(I-P) T_{F}(t)\right|_{H_{u}} \in$ $\mathcal{L}\left(H_{u}\right)$ satisfies the estimate

$$
\left\|\left.T_{u}(-t)(I-P) T_{F}(t)\right|_{H_{u}}\right\|=\left\|\left.e^{g t} T_{u}(-t)(I-P) T_{-g}^{F}(t)\right|_{H_{u}}\right\| \leq C e^{-\tau t}, \quad t \geq 0,
$$

for some constants $\tau>0$ and $C>0$, which are independent of $t$. Using the positivity of $\tau$, we can choose a $t_{0}>0$ (sufficiently large) such that $\left\|\left.T_{u}\left(-t_{0}\right)(I-P) T_{F}\left(t_{0}\right)\right|_{H_{u}}\right\|<$ 
1, and thus the operator $\left.I\right|_{H_{u}}-\left.T_{u}\left(-t_{0}\right)(I-P) T_{F}\left(t_{0}\right)\right|_{H_{u}}$ is invertible in $\mathcal{L}\left(H_{u}\right)$. Let $x_{0} \in H_{u}$ be arbitrary and define

$$
\begin{aligned}
z_{0} & :=\left[I-\left.T_{u}\left(-t_{0}\right)(I-P) T_{F}\left(t_{0}\right)\right|_{H_{u}}\right]^{-1} T_{u}\left(-t_{0}\right) x_{0}, \\
u(\rho) & :=-F T_{F}(\rho) z_{0} .
\end{aligned}
$$

Thus $u \in \boldsymbol{L}_{2}\left(0, t_{0} ; \mathbb{C}^{m}\right)$. By (7) and (15) we get

$$
\begin{aligned}
(I-P) T_{F}\left(t_{0}\right) z_{0} & =T_{u}\left(t_{0}\right) z_{0}-(I-P) \int_{0}^{t_{0}} T\left(t_{0}-\rho\right) B u(\rho) d \rho \\
& =T_{u}\left(t_{0}\right) z_{0}-\int_{0}^{t_{0}} T_{u}\left(t_{0}-\rho\right)(I-\tilde{P}) B u(\rho) d \rho,
\end{aligned}
$$

which is equivalent to

$$
T_{u}\left(t_{0}\right)\left[z_{0}-T_{u}\left(-t_{0}\right)(I-P) T_{F}\left(t_{0}\right) z_{0}\right]=\int_{0}^{t_{0}} T_{u}\left(t_{0}-\rho\right)(I-\tilde{P}) B u(\rho) d \rho
$$

and thus

$$
x_{0}=\int_{0}^{t_{0}} T_{u}\left(t_{0}-\rho\right)(I-\tilde{P}) B u(\rho) d \rho .
$$

Consequently, $\Sigma\left(A_{u},(I-\tilde{P}) B\right)$ is exactly controllable in finite time.

The following example shows that there exist optimizable systems $\Sigma(A, B)$ for which $A$ does not satisfy $\operatorname{SDA}(g)$ at any $g \leq 0$, but there is a projection $P$ that fulfills (1)-(3) of Theorem 5.1.

EXAMPLE 5.11. Let $\left\{e_{n}\right\}_{n \in \mathbb{Z}} \cup\left\{f_{n}\right\}_{n \in \mathbb{Z}}$ be an orthonormal basis of $\ell_{2},\left\{\alpha_{n}\right\}_{n \in \mathbb{Z}}=$ $\mathbb{Q} \cap[-1,0),\left\{\beta_{n}\right\}_{n \in \mathbb{Z}}=\mathbb{Q} \cap(-\infty,-1), \mu_{n}:=\alpha_{n}+i n$ and $\nu_{n}:=\beta_{n}+i n, n \in \mathbb{Z}$. We define $A: D(A) \rightarrow H$ by

$$
\begin{aligned}
A & :=\sum_{n \in \mathbb{Z}} \mu_{n}\left\langle\cdot, e_{n}\right\rangle e_{n}+\sum_{n \in \mathbb{Z}} \nu_{n}\left\langle\cdot, f_{n}\right\rangle f_{n}, \\
D(A) & :=\left\{\left.x \in H\left|\sum_{n \in \mathbb{Z}}\right| \mu_{n}\right|^{2}\left|\left\langle x, e_{n}\right\rangle\right|^{2}+\sum_{n \in \mathbb{Z}}\left|\nu_{n}\right|^{2}\left|\left\langle x, f_{n}\right\rangle\right|^{2}<\infty\right\}
\end{aligned}
$$

and $B: \mathbb{C} \rightarrow H_{-1}$ by

$$
B:=\sum_{n \in \mathbb{Z}} e_{n}
$$

Clearly, $A$ is a Riesz-spectral operator, and $A$ does not satisfy $\operatorname{SDA}(g)$ for any $g \leq 0$.

$A$ is the generator of the $C_{0}$-semigroup $T(t)$ given by

$$
T(t):=\sum_{n \in \mathbb{Z}} e^{\mu_{n} t}\left\langle\cdot, e_{n}\right\rangle e_{n}+\sum_{n \in \mathbb{Z}} e^{\nu_{n} t}\left\langle\cdot, f_{n}\right\rangle f_{n}
$$

Weiss [32] shows that $B$ is an admissible control operator for $T(t)$. It is easy to see that

$$
P z:=\sum_{n \in \mathbb{Z}}\left\langle z, f_{n}\right\rangle f_{n}
$$

is a projection operator on $H$ which commutes with $A$ and $T(t), t \geq 0$. Moreover, by [3, Theorem 2.3.5] we get that 
(1) $T_{s}(t):=P T(t)$ is an exponentially stable $C_{0}$-semigroup on $H_{s}:=\operatorname{Im}(P)$,

(2) $T_{u}(t):=(I-P) T(t)$ is a $C_{0}$-group on $H_{u}:=N(P)$,

(3) $\left\|T_{u}(t) x\right\| \leq M e^{-t}\|x\|$ for all $x \in H_{u}, t \leq 0$,

where $M>0$ is independent of $x$ and $t$. Lemma 4.4 now shows that $(I-\tilde{P}) B$ is an admissible control operator for $T_{u}(t)$.

Next we prove that $\Sigma\left(A_{u},(I-\tilde{P}) B\right)$ is exactly controllable in finite time. By Young [38, Corollary 2, p. 196] we get that $\left\{e^{\mu_{n} t}\right\}_{n \in \mathbb{Z}}$ forms a Riesz basis of $\boldsymbol{L}_{2}(-\pi, \pi)$, and thus it is easy to show that $\left\{e^{\mu_{n} t}\right\}_{n \in \mathbb{Z}}$ forms a Riesz basis for $\boldsymbol{L}_{2}(0,2 \pi)$. This implies that $\left\{g_{n}\right\}_{n \in \mathbb{Z}}, g_{n}(t):=e^{\mu_{n}(2 \pi-t)}$ is a Riesz basis for $\boldsymbol{L}_{2}(0,2 \pi)$ as well. Let $\left\{h_{n}\right\}_{n \in \mathbb{Z}}$ be the biorthogonal sequence of $\left\{g_{n}\right\}_{n \in \mathbb{Z}}$ in $\boldsymbol{L}_{2}(0,2 \pi)$ [38, p. 29], which again is a Riesz basis of $\boldsymbol{L}_{2}(0,2 \pi)\left[38\right.$, p. 36]. By definition, the biorthogonality of $\left\{g_{n}\right\}_{n \in \mathbb{Z}}$ and $\left\{h_{n}\right\}_{n \in \mathbb{Z}}$ implies that

$$
\int_{0}^{2 \pi} g_{m}(t) \overline{h_{n}(t)} d t=\delta_{n m}, \quad n, m \in \mathbb{Z}
$$

Thus we get

$$
\begin{aligned}
\int_{0}^{2 \pi} T_{u}(2 \pi-\rho) B \overline{h_{n}(\rho)} d \rho & =\int_{0}^{2 \pi} \sum_{m \in \mathbb{Z}} e^{\mu_{m}(2 \pi-\rho)} \overline{h_{n}(\rho)} e_{m} d \rho \\
& =\sum_{m \in \mathbb{Z}} \int_{0}^{2 \pi} g_{m}(\rho) \overline{h_{n}(\rho)} d \rho e_{m} \\
& =e_{n} .
\end{aligned}
$$

Let $x \in H_{u}$ be arbitrary. Then $x=\sum_{n \in \mathbb{Z}}\left\langle x, e_{n}\right\rangle e_{n}$ and $\left\{\left\langle x, e_{n}\right\rangle\right\}_{n \in \mathbb{Z}} \in \ell_{2}$. Defining $u \in \boldsymbol{L}_{2}(0,2 \pi)$ by

$$
u:=\sum_{n \in \mathbb{Z}}\left\langle x, e_{n}\right\rangle \overline{h_{n}}
$$

(19) implies that

$$
\int_{0}^{2 \pi} T(2 \pi-\rho) B u(\rho) d \rho=x .
$$

This shows that system $\Sigma\left(A_{u},(I-\tilde{P}) B\right)$ is exactly controllable in finite time. Finally, Theorem 5.1 implies that system $\Sigma(A, B)$ is optimizable.

For the proof of Theorem 5.3 we need the following lemma.

Lemma 5.12. Assume that system $\Sigma(A, B)$ is optimizable. Let $\left(s_{n}\right)_{n}$ be a sequence in $\rho(A) \cap \overline{\mathbb{C}_{0}^{+}}$and $\delta>0$ be a constant such that

$$
\left\{s \in \mathbb{C}|| s-s_{n} \mid<\delta\right\} \subset \rho(A)
$$

for every $n \in \mathbb{N}$. Then

$$
\sup _{n \in \mathbb{N}}\left\|\left(s_{n} I-A\right)^{-1} B\right\|<\infty \quad \text { and } \quad \sup _{n \in \mathbb{N}}\left\|\left(s_{n} I-A\right)^{-1}\right\|<\infty .
$$

Proof. First we prove that $\sup _{n \in \mathbb{N}}\left\|\left(s_{n} I-A\right)^{-1} B\right\|<\infty$ holds. Since the system is optimizable, there exists an LQ-stabilizing feedback $F$. Let $g_{b}^{F}$ be the (negative) closed-loop growth bound, let $\sigma \in\left(g_{b}^{F}, 0\right)$, and define $\tau:=\frac{1}{2} \min \{-\sigma, \delta\}$. If 
$\sup _{n \in \mathbb{N}}\left\|\left(s_{n} I-A\right)^{-1} B\right\|=\infty$, then the principle of uniform boundedness implies that there exist a vector $u \in \mathbb{C}^{m}$ and an element $y \in H$ such that

$$
\sup _{n \in \mathbb{N}}\left|\left\langle\left(s_{n} I-A\right)^{-1} B u, y\right\rangle\right|=\infty .
$$

Since $B$ is an admissible control operator for $T(t)$, there exists a constant $\gamma>\tau$ such that

$$
(\cdot I-A)^{-1} B \in \boldsymbol{H}_{\infty}\left(\mathbb{C}_{\gamma}^{+} ; H\right)
$$

[35, Proposition 2.3]. Since $s_{n} \in \overline{\mathbb{C}_{0}^{+}}$, we get $s_{n}+2 \gamma \in \mathbb{C}_{\gamma}^{+}$.

We write $B$ as $\left[b_{1}, \ldots, b_{m}\right]$. By Proposition 3.6, for every $n \in \mathbb{N}$ and all $k \in$ $\{1, \ldots, m\}$ there exist $\xi_{s_{n}, k} \in \boldsymbol{H}^{\infty}\left(\mathbb{C}_{\sigma}^{+} ; H\right)$ and $\omega_{s_{n}, k} \in \boldsymbol{H}^{\infty}\left(\mathbb{C}_{\sigma}^{+} ; \mathbb{C}^{m}\right)$ such that

$$
\left(\left(s_{n}+2 \gamma\right) I-A\right)^{-1} b_{k}=(s I-A) \xi_{s_{n}, k}(s)-B \omega_{s_{n}, k}(s), \quad s \in \mathbb{C}_{\sigma}^{+} .
$$

Moreover, using (22) and Proposition 3.6, the functions $\xi_{s_{n}, k}$ and $\omega_{s_{n}, k}$ can be chosen in such a way that there exists a constant $M>0$ with

$$
\left\|\xi_{s_{n}, k}\right\|_{\boldsymbol{H}_{\infty}\left(\mathbb{C}_{\sigma}^{+} ; H\right)} \leq M \quad \text { and } \quad\left\|\omega_{s_{n}, k}\right\|_{\boldsymbol{H}_{\infty}\left(\mathbb{C}_{\sigma}^{+} ; \mathbb{C}^{m}\right)} \leq M
$$

for $n \in \mathbb{N}$ and $k \in\{1, \ldots, m\}$. Defining $\Xi_{s_{n}}(s):=\left[\xi_{s_{n}, 1}(s), \ldots, \xi_{s_{n}, m}(s)\right]$ and $\Omega_{s_{n}}(s):=\left[\omega_{s_{n}, 1}(s), \ldots, \omega_{s_{n}, m}(s)\right]$, we obtain

$$
\left(\left(s_{n}+2 \gamma\right) I-A\right)^{-1} B=(s I-A) \Xi_{s_{n}}(s)-B \Omega_{s_{n}}(s), \quad s \in \mathbb{C}_{\sigma}^{+} .
$$

The functions $\Xi_{s_{n}}$ and $\Omega_{s_{n}}$ also are holomorphic on $\mathbb{C}_{\sigma}^{+}$and there exists a number $\tilde{M}>0$ with

$$
\left\|\Xi_{s_{n}}\right\|_{\boldsymbol{H}_{\infty}\left(\mathbb{C}_{\sigma}^{+} ; H^{m}\right)} \leq \tilde{M} \quad \text { and } \quad\left\|\Omega_{s_{n}}\right\|_{\boldsymbol{H}_{\infty}\left(\mathbb{C}_{\sigma}^{+} ; \mathbb{C}^{m \times m}\right)} \leq \tilde{M} .
$$

Now for $s \in \mathbb{C}_{\sigma}^{+}$, we define the functions

$$
\widetilde{\Xi}_{s_{n}}(s):=\Xi_{s_{n}}\left(s_{n}+s\right) \text { and } \widetilde{\Omega}_{s_{n}}(s):=\Omega_{s_{n}}\left(s_{n}+s\right) .
$$

Since $\operatorname{Re}\left(s_{n}\right) \geq 0$, the functions $\widetilde{\Xi}_{s_{n}}$ and $\widetilde{\Omega}_{s_{n}}$ also are holomorphic and bounded (with the same estimates) on $\mathbb{C}_{\sigma}^{+}$. Multiplying equation (23) (with $s_{n}+s$ instead of $s$ ) by $\left(\left(s_{n}+s\right) I-A\right)^{-1},|s|<\tau$, and using the resolvent identity, we get

$$
\left(\left(s_{n}+s\right) I-A\right)^{-1} B\left[\widetilde{\Omega}_{s_{n}}(s)-\frac{I}{s-2 \gamma}\right]=\widetilde{\Xi}_{s_{n}}(s)-\frac{1}{s-2 \gamma}\left(\left(s_{n}+2 \gamma\right) I-A\right)^{-1} B
$$

Note that the existence of $\left(\left(s_{n}+s\right) I-A\right)^{-1},|s|<\tau$, is guaranteed by (20). Since $\left\{\widetilde{\Omega}_{s_{n}}\right\}_{n}$ is a uniformly bounded set in $\boldsymbol{H}_{\infty}\left(\mathbb{C}_{\sigma}^{+} ; \mathbb{C}^{m \times m}\right)$, there exists a subsequence $\left\{\widetilde{\Omega}_{s_{n_{j}}}\right\}_{j}$ of $\left\{\widetilde{\Omega}_{s_{n}}\right\}_{n}$ which converges uniformly on compact subsets of $\mathbb{C}_{\sigma}^{+}$to a holomorphic function $\widetilde{\Omega} \in \boldsymbol{H}_{\infty}\left(\mathbb{C}_{\sigma}^{+} ; \mathbb{C}^{m \times m}\right)$ (see Hille and Phillips [13, Theorem 3.14.2]). We denote this subsequence again by $\left\{\widetilde{\Omega}_{s_{n}}\right\}_{n}$.

For $\beta \in(0, \tau]$ we define $B(\beta):=\{s \in \mathbb{C}:|s| \leq \beta\}$. We now prove that there exists a number $\tilde{\tau} \in(0, \tau]$ such that

$$
\operatorname{det}\left(\widetilde{\Omega}(s)-\frac{I}{s-2 \gamma}\right) \neq 0
$$


for all $s \in B(\tilde{\tau}) \backslash\{0\}$. If it were not true, then there would exist a sequence $\left(\delta_{n}\right)_{n} \subset$ $B(\tau) \backslash\{0\}$, tending to 0 as $n$ tends to $\infty$, such that

$$
\operatorname{det}\left(\widetilde{\Omega}\left(\delta_{n}\right)-\frac{I}{\delta_{n}-2 \gamma}\right)=0, \quad n \in \mathbb{N} .
$$

Now

$$
\operatorname{det}\left(\widetilde{\Omega}\left(\delta_{n}\right)-\frac{I}{\delta_{n}-2 \gamma}\right)=\operatorname{det}\left(\left(\delta_{n}-2 \gamma\right) \widetilde{\Omega}\left(\delta_{n}\right)-I\right) \operatorname{det}\left(\frac{I}{\delta_{n}-2 \gamma}\right)
$$

implies that

$$
\operatorname{det}\left(\left(\delta_{n}-2 \gamma\right) \widetilde{\Omega}\left(\delta_{n}\right)-I\right)=0, \quad n \in \mathbb{N}
$$

In other words, $\operatorname{det}((s-2 \gamma) \widetilde{\Omega}(s)-I)$ would have a converging sequence of zeros in $B(\tau)$. By the holomorphicity of $\widetilde{\Omega}$ on $\mathbb{C}_{\sigma}^{+}$and of the determinant on $\mathbb{C}^{m \times m}$, we get that $\operatorname{det}((s-2 \gamma) \tilde{\Omega}(s)-I)$ is holomorphic on $\mathbb{C}_{\sigma}^{+}$. Since $-\tau>\sigma$, this would imply that the function $\operatorname{det}((s-2 \gamma) \widetilde{\Omega}(s)-I)$ would be equal to zero everywhere on $\mathbb{C}_{\sigma}^{+}$. Taking $s=2 \gamma\left(\right.$ note that $\left.2 \gamma \in \mathbb{C}_{\sigma}^{+}\right)$, we get a contradiction. Therefore, there exists a number $\tilde{\tau} \in(0, \tau]$ such that

$$
\operatorname{det}\left(\widetilde{\Omega}(s)-\frac{I}{s-2 \gamma}\right) \neq 0 \quad \text { for all } s \in B(\tilde{\tau}) \backslash\{0\} .
$$

On $B(\tilde{\tau})$ we now define the functions

$$
f_{n}(s):=\left\langle\left(\left(s+s_{n}\right) I-A\right)^{-1} B u, y\right\rangle, \quad n \in \mathbb{N},
$$

where $u$ and $y$ are the vectors from (21). Since $\tilde{\tau}<\delta$, we get by (20) that $f_{n}$ is holomorphic, and so by the maximum principle there exists a number $\theta_{n} \in[0,2 \pi)$ such that

$$
\left|f_{n}\left(\tilde{\tau} e^{i \theta_{n}}\right)\right| \geq\left|f_{n}(0)\right| .
$$

Since (by (21)) $\sup _{n \in \mathbb{N}}\left|f_{n}(0)\right|=\infty$, equation (27) implies

$$
\sup _{n \in \mathbb{N}}\left|f_{n}\left(\tilde{\tau} e^{i \theta_{n}}\right)\right|=\infty .
$$

There now exists a subsequence of $\left\{\theta_{n}\right\}_{n=1}^{\infty}$ which converges to $\theta \in[0,2 \pi]$. Again we rename the subsequence $\left\{\theta_{n}\right\}_{n=1}^{\infty}$. By the choice of $\tilde{\tau}$, we have that

$$
\operatorname{det}\left(\widetilde{\Omega}\left(\tilde{\tau} e^{i \theta}\right)-\frac{I}{\tilde{\tau} e^{i \theta}-2 \gamma}\right) \neq 0 .
$$

The set of invertible matrices is open and $\widetilde{\Omega}_{s_{n}}\left(\tilde{\tau} e^{i \theta_{n}}\right)-\frac{I}{\tilde{\tau} e^{i \theta_{n}}-2 \gamma}$ converges to $\widetilde{\Omega}\left(\tilde{\tau} e^{i \theta}\right)-$ $\frac{I}{\tilde{\tau} e^{i \theta}-2 \gamma}$. Thus there exists a number $N \in \mathbb{N}$ such that

$$
\operatorname{det}\left(\widetilde{\Omega}_{s_{n}}\left(\tilde{\tau} e^{i \theta_{n}}\right)-\frac{I}{\tilde{\tau} e^{i \theta_{n}}-2 \gamma}\right) \neq 0, \quad n \geq N
$$


Multiplying (25) (with $\left.s:=\tilde{\tau} e^{i \theta_{n}}\right)$ by $\left[\widetilde{\Omega}_{s_{n}}\left(\tilde{\tau} e^{i \theta_{n}}\right)-\frac{I}{\tilde{\tau} e^{i \theta_{n}}-2 \gamma}\right]^{-1} u$ and taking the inner product with $y$, we obtain

$$
\begin{gathered}
f_{n}\left(\tilde{\tau} e^{i \theta_{n}}\right)=\left\langle\left[\widetilde{\Xi}_{s_{n}}\left(\tilde{\tau} e^{i \theta_{n}}\right)-\frac{1}{\tilde{\tau} e^{i \theta_{n}}-2 \gamma}\left(\left(s_{n}+2 \gamma\right) I-A\right)^{-1} B\right]\right. \\
\left.\cdot\left[\widetilde{\Omega}_{s_{n}}\left(\tilde{\tau} e^{i \theta_{n}}\right)-\frac{I}{\tilde{\tau} e^{i \theta_{n}}-2 \gamma}\right]^{-1} u, y\right\rangle .
\end{gathered}
$$

Now $\left[\widetilde{\Omega}_{s_{n}}\left(\tilde{\tau} e^{i \theta_{n}}\right)-\frac{I}{\tilde{\tau} e^{i \theta_{n}}-2 \gamma}\right]^{-1}$ converges to $\left[\widetilde{\Omega}\left(\tilde{\tau} e^{i \theta}\right)-\frac{I}{\tilde{\tau} e^{i \theta}-2 \gamma}\right]^{-1}$. Thus using (22) and (24), we see that the right-hand side of (29) is bounded. However, this is in contradiction with (28) and so

$$
\sup _{n \in \mathbb{N}}\left\|\left(s_{n} I-A\right)^{-1} B\right\|<\infty .
$$

It now remains to prove that $\sup _{n \in \mathbb{N}}\left\|\left(s_{n} I-A\right)^{-1}\right\|<\infty$. Assume that $\sup _{n \in \mathbb{N}} \|\left(s_{n} I-\right.$ $A)^{-1} \|=\infty$. By the principle of uniform boundedness there exists a $z \in H$ such that

$$
\sup _{n \in \mathbb{N}}\left\|\left(s_{n} I-A\right)^{-1} z\right\|=\infty .
$$

By Proposition 3.6 there exist $\omega_{z} \in \boldsymbol{H}_{\infty}\left(\mathbb{C}_{\sigma}^{+} ; \mathbb{C}^{m}\right)$ and $\xi_{z} \in \boldsymbol{H}_{\infty}\left(\mathbb{C}_{\sigma}^{+} ; H\right)$ such that we have

$$
\xi_{z}\left(s_{n}\right)=\left(s_{n} I-A\right)^{-1} z+\left(s_{n} I-A\right)^{-1} B \omega_{z}\left(s_{n}\right), \quad n \in \mathbb{N} .
$$

Since $\left\{\left\|\xi_{z}\left(s_{n}\right)\right\|\right\}_{n}$ and $\left\{\left\|\omega_{z}\left(s_{n}\right)\right\|\right\}_{n}$ are bounded sets, it follows with (31) that

$$
\sup _{n \in \mathbb{N}}\left\|\left(s_{n} I-A\right)^{-1} B\right\|=\infty
$$

which is in contradiction with (30). This completes the proof.

Corollary 5.13. Assume that $\Sigma(A, B)$ is optimizable and let $A$ satisfy the SDA. Then

$$
(\cdot I-A)^{-1} \in \boldsymbol{L}_{\infty}(i \mathbb{R} ; \mathcal{L}(H)) \quad \text { and } \quad(\cdot I-A)^{-1} B \in \boldsymbol{L}_{\infty}\left(i \mathbb{R} ; \mathcal{L}\left(\mathbb{C}^{m}, H\right)\right) .
$$

Proof. Since $A$ satisfies the SDA, there exists a $\delta>0$ such that $\mathbb{C}_{-\delta}^{+} \cap \mathbb{C}_{\delta}^{-} \subset \rho(A)$. Now it is easy to see that $(\cdot I-A)^{-1}: i \mathbb{R} \rightarrow \mathcal{L}(H)$ and $(\cdot I-A)^{-1} B: i \mathbb{R} \rightarrow \mathcal{L}\left(\mathbb{C}^{m}, H\right)$ are continuous. If the statement does not hold, then there exists a sequence $\left(s_{n}\right)_{n} \in i \mathbb{R}$ such that

$$
\lim _{n \rightarrow \infty}\left\|\left(s_{n} I-A\right)^{-1}\right\|=\infty \quad \text { or } \quad \lim _{n \rightarrow \infty}\left\|\left(s_{n} I-A\right)^{-1} B\right\|=\infty,
$$

which is in contradiction with Lemma 5.12.

By $g_{\sigma_{p}}(A)$ we denote the bound for the point spectrum of $A$, i.e.,

$$
g_{\sigma_{p}}(A):=\sup \left\{\operatorname{Re}(\lambda) \mid \lambda \in \sigma_{p}(A)\right\} .
$$

It is easy to see that $g_{\sigma_{p}}(A) \leq g_{b}(T)$. The following theorem shows that for an optimizable system $\Sigma(A, B)$ we cannot have $g_{\sigma_{p}}(A)<0 \leq g_{b}(T)$. For one-dimensional control operators this result also can be found in [28]. 
Theorem 5.14. Assume that $\Sigma(A, B)$ is optimizable and that $\sigma_{p}(A) \subset \mathbb{C}_{-\tau}^{-}$for some $\tau>0$. Then $T(t)$ is exponentially stable.

Proof. If $T(t)$ is not exponentially stable, then Huang [14] shows that $\left\|(s I-A)^{-1}\right\|$ is not bounded in $\mathbb{C}_{0}^{+}$. Therefore, there exists a sequence $\left(s_{n}\right)_{n} \subset \mathbb{C}_{0}^{+}$such that

$$
\lim _{n \rightarrow \infty}\left\|\left(s_{n} I-A\right)^{-1}\right\|=\infty .
$$

Since $\Sigma(A, B)$ is optimizable, by Theorem 3.3 there exists an LQ-stabilizing feedback $F$. By Proposition 3.5 we get that $\sigma_{g_{b}^{F}}^{+}(A)$ consists of only point spectrum. Thus, for $-\delta:=\max \left\{-\tau, g_{b}^{F}\right\}$ we obtain $\sigma_{-\delta}^{+}(A)=\emptyset$. From the fact $\sigma_{-\delta}^{+}(A)=\emptyset$, we see that the sequence $\left(s_{n}\right)_{n}$ satisfies equation (20). Hence equation (32) is in contradiction with the result of Lemma 5.12.

REMARK 5.15. There are many cases where a generator satisfies $g_{\sigma_{p}}(A)<0$ and $g_{b}(T) \geq 0$. This can happen, for example, when the spectrum consists solely of eigenvalues, but the multiplicity of the eigenvalues is not bounded (see Zabczyk [39]) or for certain shift semigroups (see Greiner, Voigt, and Wolff [11] or Curtain and Zwart [3, Example 5.1.4]). This means that for such generators $A$ there exists no admissible finite-rank control operator $B$ for $T(t)$ such that the system $\Sigma(A, B)$ is optimizable.

We are now going to prove the main result of this section.

Proof of Theorem 5.3. Since $\Sigma(A, B)$ is optimizable, we have from Theorem 3.3 the existence of an LQ-stabilizing feedback $F$. Let $T_{F}(t)$ be as in Definition 3.2, and let $g_{b}^{F}$ be the (negative) growth bound of $T_{F}(t)$. By the SDA we have the existence of $g_{1}<0<g_{2}$ such that (18) holds. Since (18) remains valid if we increase $g_{1}\left(g_{1}\right.$ must remain negative), without loss of generality we may assume that $g_{b}^{F}<g_{1}$ holds. Let $g \in\left(g_{1}, g_{2}\right)$ be arbitrary. By Theorem 3.3 and Proposition 3.4 the system $\Sigma(A-g I, B)$ is optimizable. The choice of $g$ implies the existence of $\delta>0$ such that

$$
\mathbb{C}_{-\delta}^{+} \cap \mathbb{C}_{\delta}^{-} \subset \rho(A-g I),
$$

and thus Corollary 5.13 shows

$$
((\cdot+g) I-A)^{-1} \in \boldsymbol{L}_{\infty}(i \mathbb{R} ; \mathcal{L}(H)) .
$$

Therefore Prüss [24, Corollary 5] shows that

$$
\left\{\lambda \in \mathbb{C}|| \lambda \mid=e^{g}\right\} \subset \rho(T(1))
$$

holds. Since $g \in\left(g_{1}, g_{2}\right)$ is arbitrary, this implies

$$
\left\{\lambda \in \mathbb{C}\left|e^{g_{1}}<\right| \lambda \mid<e^{g_{2}}\right\} \subset \rho(T(1)) .
$$

Let $c \in\left(g_{1}, g_{2}\right)$ be arbitrary and define $P \in \mathcal{L}(H)$ by

$$
P:=\int_{|s|=e^{c}}(s I-T(1))^{-1} d s .
$$

It is easy to see that $P^{2}=P$ holds, i.e., $P$ is a projection, and that $P$ commutes with the $C_{0}$-semigroup $T(t)$. Thus part (1) is satisfied. Define $H_{s}:=\operatorname{Im}(P)$ and $H_{u}:=N(P)$. Then the spectrum of the $C_{0}$-semigroup $T_{s}(t):=P T(t)$ on $H_{s}$ satisfies

$$
\sigma\left(T_{s}(1)\right) \subset\left\{\lambda \in \mathbb{C}|| \lambda \mid<e^{g_{1}}\right\},
$$


and thus we get that the spectral radius of $T_{s}(1)$ is less than 1 . Therefore, $T_{s}(t)$ is an exponentially stable $C_{0}$-semigroup on $H_{s}$, which proves part 2 . Similarly, the spectrum of the $C_{0}$-semigroup $T_{u}(t):=(I-P) T(t)$ on $H_{u}$ satisfies

$$
\sigma\left(T_{u}(1)\right) \subset\left\{\lambda \in \mathbb{C}\left|e^{g_{2}}<\right| \lambda \mid\right\},
$$

and thus $0 \notin \sigma\left(T_{u}(1)\right)$. Then Pazy [22, Theorem 6.5] shows that $T_{u}(t)$ can be embedded in a $C_{0}$-group, and so part 3 follows. Since

$$
\sigma\left(T_{u}(-1)\right)=\sigma\left(\left(T_{u}(1)\right)^{-1}\right) \subset\left\{\lambda \in \mathbb{C}|| \lambda \mid<e^{-g_{2}}\right\},
$$

we get that the spectral radius of $T_{u}(-1)$ is less than 1 . Thus $-A_{u}$ generates an exponentially stable $C_{0}$-semigroup on $H_{u}$, and so part 4 follows.

Lemma 4.4 now shows that $(I-\tilde{P}) B$ is an admissible control operator for $T_{u}(t)$. Thus it remains to prove that $\Sigma\left(A_{u},(I-\tilde{P}) B\right)$ is exactly controllable in finite time. Since $T_{F}(t)$ and $T_{u}(-t)$ are exponentially stable, the operator $\left.T_{u}(-t)(I-P) T_{F}(t)\right|_{H_{u}}$ $\in \mathcal{L}\left(H_{u}\right)$ satisfies the estimate

$$
\left\|\left.T_{u}(-t)(I-P) T_{F}(t)\right|_{H_{u}}\right\| \leq C e^{-\tau t}, \quad t \geq 0,
$$

for some $C, \tau>0$. The proof that $\Sigma\left(A_{u},(I-\tilde{P}) B\right)$ is exactly controllable in finite time now exactly follows the proof of Theorem 5.10.

6. More information on the spectrum of $\boldsymbol{A}$. In this section, we show that the spectrum of the generator $A$ has to be of a special form for the system $\Sigma(A, B)$ to be optimizable. In Proposition 3.5 we saw already that for any LQ-stabilizing feedback $F$, the spectrum of $A$ in the right-half plane $\mathbb{C}_{g_{b}^{F}}^{+}$consists only of point spectrum with finite multiplicity and contains no finite accumulation point. We now prove that the eigenvalues cannot go too slowly to infinity if the system $\Sigma(A, B)$ is optimizable.

Let $\lambda_{0}$ be an isolated point of $\sigma(A)$ and an eigenvalue of $A$. Then $P_{\lambda_{0}} \in \mathcal{L}(H)$, given by

$$
P_{\lambda_{0}} x:=\frac{1}{2 \pi i} \int_{\Gamma}(\lambda I-A)^{-1} x d \lambda
$$

where $\Gamma$ is a simple closed contour in $\mathbb{C}$ with $\lambda_{0}$ the only point of $\sigma(A)$ in its interior and no points of $\sigma(A)$ on $\Gamma$, is a projection onto the spectral subspace corresponding to $\lambda_{0}$; see [3, Lemma 2.5.7]. The dimension of $P_{\lambda_{0}} H$ is called the algebraic multiplicity, denoted by $m_{a}\left(\lambda_{0}, A\right)$, and the geometric multiplicity is given by $m_{g}\left(\lambda_{0}, A\right):=\operatorname{dim}\left(N\left(\lambda_{0} I-A\right)\right)$. If there exists a number $p\left(\lambda_{0}, A\right)>0$ such that $\left(A-\lambda_{0} I\right)^{p\left(\lambda_{0}, A\right)-1} P_{\lambda_{0}} \neq 0$, while $\left(A-\lambda_{0} I\right)^{p} P_{\lambda_{0}}=0$ for all $p \geq p\left(\lambda_{0}, A\right)$, then the point $\lambda_{0}$ is called pole of $(\cdot I-A)^{-1}$ of order $p\left(\lambda_{0}, A\right)$. In $[1$, p. 73] it is shown that

$$
\max \left\{m_{g}\left(\lambda_{0}, A\right), p\left(\lambda_{0}, A\right)\right\} \leq m_{a}\left(\lambda_{0}, A\right) \leq p\left(\lambda_{0}, A\right) m_{g}\left(\lambda_{0}, A\right)
$$

holds. The main result of this section is the following theorem.

THEOREM 6.1. Assume that the system $\Sigma(A, B)$ is optimizable. Then for any $L Q$-stabilizing feedback $F$, the spectral subset $\sigma_{g_{b}^{F}}^{+}(A)=\left\{\lambda_{n}\right\}_{n \in \mathbb{N}}$ satisfies

$$
m_{g}\left(\lambda_{n}, A\right) \leq m, \quad n \in \mathbb{N},
$$


where $m$ is the dimension of the input space, and for every $g>g_{b}^{F}$ we have

$$
\sum_{n \in \mathbb{N}, \operatorname{Re}\left(\lambda_{n}\right)>g} \frac{m_{a}\left(\lambda_{n}, A\right)}{\left|\lambda_{n}-g\right|^{2}}<\infty .
$$

Moreover, the finite-dimensional systems $\Sigma\left(\left.A\right|_{P_{\lambda_{n}} H}, \tilde{P}_{\lambda_{n}} B\right)$ are controllable.

In [7], Fattorini proved a result that is similar to equation (34). He considers systems $\Sigma(A, B)$ with bounded control operators and assumes that there exists a nonzero holomorphic function $f$, being the Laplace transform of a function with compact support, such that for every $x \in \operatorname{Im}(f(A))$ there exists an input $u$, which may be a $\sigma$-additive measure with compact support, such that

$$
x=\int_{0}^{t} T(t-\rho) B u(d \rho) .
$$

In this situation Fattorini proved that

$$
\sum_{\lambda \in \sigma(A)} \frac{m_{a}(\lambda, A) \operatorname{Re}(\lambda)}{1+|\lambda|^{2}}<\infty .
$$

For the proof of Theorem 6.1 we need the following lemma.

Lemma 6.2. Let $\lambda$ be an isolated point of $\sigma(A)$ and an eigenvalue of $A$. Then $\bar{\lambda}$ is an isolated point of $\sigma\left(A^{*}\right)$ and an eigenvalue of $A^{*}$ with $m_{g}\left(\bar{\lambda}, A^{*}\right)=m_{g}(\lambda, A)$, $m_{a}\left(\bar{\lambda}, A^{*}\right)=m_{a}(\lambda, A)$, and $p\left(\bar{\lambda}, A^{*}\right)=p(\lambda, A)$.

Proof. The proof follows directly from Kato [18, Remark III 6.23].

Proof of Theorem 6.1. In Proposition 3.5 it is shown that $\sigma_{g_{b}^{F}}^{+}(A)$ consists only of point spectrum with finite multiplicity and contains no finite accumulation point in $\sigma_{g_{b}^{F}}^{+}(A)$. Thus we can write $\sigma_{g_{b}^{F}}^{+}(A)$ as $\left\{\lambda_{n}\right\}_{n \in \mathbb{N}}$. That the finite-dimensional systems $\Sigma\left(\left.A\right|_{P_{\lambda_{n}} H}, \tilde{P}_{\lambda_{n}} B\right)$ are controllable follows directly from [28].

1. We prove that $m_{g}\left(\lambda_{n}, A\right) \leq m$ for all $n \in \mathbb{N}$. Let $n \in \mathbb{N}$. By Proposition 3.6, for every $x_{0} \in H$ there exist a vector $\xi \in H$ and $\omega \in \mathbb{C}^{m}$ such that

$$
x_{0}=\left(\lambda_{n} I-A\right) \xi-B \omega .
$$

Since $\tilde{P}_{\lambda_{n}}$ commutes with $A$, this implies

$$
P_{\lambda_{n}} x_{0}=\left(\lambda_{n} I-A\right) P_{\lambda_{n}} \xi-\tilde{P}_{\lambda_{n}} B \omega
$$

and therefore $\left(\lambda_{n} I-A\right) P_{\lambda_{n}} H+\tilde{P}_{\lambda_{n}} B \mathbb{C}^{m} \supset P_{\lambda_{n}} H$. Thus we have

$$
\begin{aligned}
m_{a}\left(\lambda_{n}, A\right) & =\operatorname{dim}\left(P_{\lambda_{n}} H\right) \\
& \leq \operatorname{dim}\left(\left(\lambda_{n} I-A\right) P_{\lambda_{n}} H\right)+\operatorname{dim}\left(\tilde{P}_{\lambda_{n}} B \mathbb{C}^{m}\right) \\
& \leq m_{a}\left(\lambda_{n}, A\right)-m_{g}\left(\lambda_{n}, A\right)+m,
\end{aligned}
$$

which shows $m_{g}\left(\lambda_{n}, A\right) \leq m$.

2. We prove

$$
\sum_{n \in \mathbb{N}, \operatorname{Re}\left(\lambda_{n}\right)>g} \frac{m_{a}\left(\lambda_{n}, A\right)\left(\operatorname{Re}\left(\lambda_{n}\right)-g\right)}{1+\left|\lambda_{n}-g\right|^{2}}<\infty
$$


for every $g>g_{b}^{F}$. Let $g>g_{b}^{F}$ and $\tau \in\left(g_{b}^{F}, g\right)$. Then there exists $\alpha \in \rho(A)$ with $\operatorname{Re}(\alpha) \in(\tau, g)$. We write $B$ as $B=\left[b_{1}, \ldots, b_{m}\right]$. By Proposition 3.6, for every $k \in\{1, \ldots, m\}$ there exist $\xi_{k} \in \boldsymbol{H}_{\infty}\left(\mathbb{C}_{\tau}^{+} ; H\right)$ and $\omega_{k} \in \boldsymbol{H}_{\infty}\left(\mathbb{C}_{\tau}^{+} ; \mathbb{C}^{m}\right)$ such that

$$
(\alpha I-A)^{-1} b_{k}=(s I-A) \xi_{k}(s)-B \omega_{k}(s), \quad s \in \mathbb{C}_{\tau}^{+} .
$$

Defining $\Xi(s):=\left[\xi_{1}(s), \ldots, \xi_{m}(s)\right]$ and $\Omega(s):=\left[\omega_{1}(s), \ldots, \omega_{m}(s)\right]$ we obtain

$$
(\alpha I-A)^{-1} B=(s I-A) \Xi(s)-B \Omega(s), \quad s \in \mathbb{C}_{\tau}^{+} .
$$

The functions $\Xi$ and $\Omega$ also are holomorphic and bounded on $\mathbb{C}_{\tau}^{+}$.

Multiplying (35) by $(s I-A)^{-1}$ and using the resolvent identity, we obtain

$$
\frac{1}{s-\alpha}(\alpha I-A)^{-1} B=\Xi(s)-(s I-A)^{-1} B\left[\Omega(s)-\frac{1}{s-\alpha} I\right], \quad s \in \mathbb{C}_{g}^{+} \backslash \sigma(A) .
$$

Since the term on the left-hand side and the first term of the right-hand side are holomorphic on $\mathbb{C}_{g}^{+}$, the function $(s I-A)^{-1} B\left[\Omega(s)-\frac{1}{s-\alpha} I\right]$ also is holomorphic on $\mathbb{C}_{g}^{+}$. Defining

$$
F(s):=\left[\Omega(s)-\frac{1}{s-\alpha} I\right], \quad s \in \mathbb{C}_{g}^{+},
$$

it is easy to see that $F(s)^{-1} \operatorname{det}(F(s))$ is holomorphic on $\mathbb{C}_{g}^{+}$. Thus

$$
\begin{aligned}
G(s) & :=(s I-A)^{-1} B \operatorname{det}(F(s)) \\
& =(s I-A)^{-1} B\left[\Omega(s)-\frac{1}{s-\alpha} I\right] \cdot F(s)^{-1} \operatorname{det}(F(s))
\end{aligned}
$$

is holomorphic on $\mathbb{C}_{g}^{+}$.

Next we show that every $\lambda \in \sigma_{g}^{+}(A)$ is a zero of $\operatorname{det}(F(s))$ with order at least $p(\lambda, A)$. Let us assume that this is not true. Then there is one $\lambda_{0} \in \sigma_{g}^{+}(A)$ such that $\lambda_{0}$ is no zero of $\operatorname{det}(F(s))$ or $\lambda_{0}$ is a zero of $\operatorname{det}(F(s))$, but the order of $\lambda_{0}$ as zero of $\operatorname{det}(F(s))$ is less than $p:=p\left(\lambda_{0}, A\right)$. By $f^{(i)}$ we denote the $i$ th derivative of $f$. Let $k_{0} \in\{0, \ldots, p-1\}$ be such that $\operatorname{det}\left(F\left(\lambda_{0}\right)\right)^{(i)}=0$, $i=0, \ldots, k_{0}-1$, while $\operatorname{det}\left(F\left(\lambda_{0}\right)\right)^{\left(k_{0}\right)} \neq 0$ and let $\delta>0$ be such that $B\left(\lambda_{0}\right):=\left\{s \in \mathbb{C}|0<| s-\lambda_{0} \mid<\delta\right\} \subset \rho(A)$. Since $\tilde{P}_{\lambda_{0}}$ commutes with the resolvent $(\cdot I-A)^{-1}$, by Lemma 4.2 we obtain that

$$
\begin{aligned}
G_{\lambda_{0}}(s) & :=P_{\lambda_{0}} G(s) \\
& =\left(s I-\left.A\right|_{P_{\lambda_{0}} H}\right)^{-1} \tilde{P}_{\lambda_{0}} B \operatorname{det}(F(s))
\end{aligned}
$$

is holomorphic on $\mathbb{C}_{g}^{+}$. Since $\left(s I-\left.A\right|_{P_{\lambda_{0}} H}\right)^{-1}=(s I-A)^{-1}$ on $P_{\lambda_{0}} H$ (see Lemma 4.2), we get $p=p\left(\lambda_{0},\left.A\right|_{P_{\lambda_{0}} H}\right)$. Expressing $\left(\cdot I-\left.A\right|_{P_{\lambda_{0}} H}\right)^{-1}$ in its Laurent series, we obtain

$$
G_{\lambda_{0}}(s)=\tilde{G}_{\lambda_{0}}(s)+\sum_{k=0}^{p-1} \frac{\operatorname{det}(F(s))}{\left(s-\lambda_{0}\right)^{k+1}}\left(\lambda_{0} I-\left.A\right|_{P_{\lambda_{0}} H}\right)^{k} \tilde{P}_{\lambda_{0}} B, \quad s \in B\left(\lambda_{0}\right),
$$


where $\tilde{G}_{\lambda_{0}}(s)$ is a holomorphic function on $B\left(\lambda_{0}\right) \cup\left\{\lambda_{0}\right\}$. Since $\lambda_{0}$ is a zero of $\operatorname{det}(F(s))$ with order $k_{0}$, we get

$$
\sum_{k=0}^{k_{0}-1} \frac{\operatorname{det}(F(s))}{\left(s-\lambda_{0}\right)^{k+1}}\left(\lambda_{0} I-\left.A\right|_{P_{\lambda_{0}} H}\right)^{k} \tilde{P}_{\lambda_{0}} B
$$

is holomorphic at $\lambda_{0}$. Since $G_{\lambda_{0}}(s)$ is holomorphic at $\lambda_{0}$, this would imply that

$$
\sum_{k=k_{0}}^{p-1} \frac{\operatorname{det}(F(s))}{\left(s-\lambda_{0}\right)^{k+1}}\left(\lambda_{0} I-\left.A\right|_{P_{\lambda_{0}} H}\right)^{k} \tilde{P}_{\lambda_{0}} B
$$

is holomorphic on $\mathbb{C}_{g}^{+}$. Thus using the definition of a pole, we get that

$$
\begin{aligned}
& \left(\lambda_{0} I-\left.A\right|_{P_{\lambda_{0}} H}\right)^{p-k_{0}-1} \sum_{k=k_{0}}^{p-1} \frac{\operatorname{det}(F(s))}{\left(s-\lambda_{0}\right)^{k+1}}\left(\lambda_{0} I-\left.A\right|_{P_{\lambda_{0}} H}\right)^{k} \tilde{P}_{\lambda_{0}} B \\
& =\frac{\operatorname{det}(F(s))}{\left(s-\lambda_{0}\right)^{k_{0}+1}}\left(\lambda_{0} I-\left.A\right|_{P_{\lambda_{0}} H}\right)^{p-1} \tilde{P}_{\lambda_{0}} B
\end{aligned}
$$

is holomorphic at $\lambda_{0}$. However, this can only happen if $\left(\lambda_{0} I-\left.A\right|_{P_{\lambda_{0}} H}\right)^{p-1} \tilde{P}_{\lambda_{0}} B$ $=0$. By the definition of a pole there is a $x \in P_{\lambda_{0}} H$ such that $\left(\lambda_{0} I-\right.$ $\left.\left.A\right|_{P_{\lambda_{0}} H}\right)^{p-1} x \neq 0$. The optimizability of system $\Sigma(A, B)$ implies that there are elements $y \in H$ and $u \in \mathbb{C}^{m}$ such that

$$
x=\left(\lambda_{0} I-A\right) y-B u .
$$

Since $x \in P_{\lambda_{0}} H$, this implies $x=\left(\lambda_{0} I-\left.A\right|_{P_{\lambda_{0}} H}\right) P_{\lambda_{0}} y-\tilde{P}_{\lambda_{0}} B u$. Multiplying both sides with $\left(\lambda_{0} I-\left.A\right|_{P_{\lambda_{0}} H}\right)^{p-1}$, we get

$0 \neq\left(\lambda_{0} I-\left.A\right|_{P_{\lambda_{0}} H}\right)^{p-1} x=\left(\lambda_{0} I-\left.A\right|_{P_{\lambda_{0}} H}\right)^{p} y-\left(\lambda_{0} I-\left.A\right|_{P_{\lambda_{0}} H}\right)^{p-1} \tilde{P}_{\lambda_{0}} B u=0$,

which is a contradiction. Thus

$$
f(s):=\operatorname{det}(F(s)), \quad s \in \mathbb{C}_{g}^{+},
$$

is holomorphic and bounded on $\mathbb{C}_{g}^{+}$and every $\lambda \in \mathbb{C}_{g}^{+} \cap \sigma(A)$ is a zero of $f$ with order at least $p(\lambda, A)$.

Next we show that $f$ is not identically zero. Assume that $f$ is identical to zero on $\mathbb{C}_{g}^{+}$. Using (36), (37),

$$
f(s)=\operatorname{det}((s-\alpha) \Omega(s)-I) \operatorname{det}\left(\frac{1}{s-\alpha} I\right), \quad s \in \mathbb{C}_{g}^{+},
$$

it would imply that

$$
\operatorname{det}((s-\alpha) \Omega(s)-I)=0, \quad s \in \mathbb{C}_{g}^{+} .
$$

Now the holomorphicity of $\operatorname{det}((s-\alpha) \Omega(s)-I)$ on $\mathbb{C}_{\tau}^{+}$implies

$$
0=\operatorname{det}((\alpha-\alpha) \Omega(\alpha)-I)=\operatorname{det}(I)=1,
$$


which is a contradiction. Thus $f \not \equiv 0$ on $\mathbb{C}_{g}^{+}$.

Applying now Duren [6, Theorem 11.3] to $f \in \boldsymbol{H}_{\infty}\left(\mathbb{C}_{g}^{+}\right)$shows

$$
\sum_{n \in \mathbb{N}, \operatorname{Re}\left(\lambda_{n}\right)>g} \frac{p\left(\lambda_{n}, A\right)\left(\operatorname{Re}\left(\lambda_{n}\right)-g\right)}{1+\left|\lambda_{n}-g\right|^{2}}<\infty .
$$

Therefore, using (33) and $m_{g}\left(\lambda_{n}, A\right) \leq m$, we get

$$
\sum_{n \in \mathbb{N}, \operatorname{Re}\left(\lambda_{n}\right)>g} \frac{m_{a}\left(\lambda_{n}, A\right)\left(\operatorname{Re}\left(\lambda_{n}\right)-g\right)}{1+\left|\lambda_{n}-g\right|^{2}}<\infty .
$$

3. It remains to prove that

$$
\sum_{n \in \mathbb{N}, \operatorname{Re}\left(\lambda_{n}\right)>g} \frac{m_{a}\left(\lambda_{n}, A\right)}{\left|\lambda_{n}-g\right|^{2}}<\infty
$$

for every $g>g_{b}^{F}$. Let $k_{n}:=m_{a}\left(\lambda_{n}, A\right), g>g_{b}^{F}$ and $\tilde{g} \in\left(g_{b}^{F}, g\right)$. By part (2), we have

$$
\sum_{n \in \mathbb{N}, \operatorname{Re}\left(\lambda_{n}\right)>g} \frac{k_{n}\left(\operatorname{Re}\left(\lambda_{n}\right)-g\right)}{1+\left|\lambda_{n}-g\right|^{2}}<\infty \quad \text { and } \sum_{n \in \mathbb{N}, \operatorname{Re}\left(\lambda_{n}\right)>g} \frac{k_{n}\left(\operatorname{Re}\left(\lambda_{n}\right)-\tilde{g}\right)}{1+\left|\lambda_{n}-\tilde{g}\right|^{2}}<\infty .
$$

For any $\lambda \in \mathbb{C}$ with $|\lambda-g| \geq 1$ we get

$$
\begin{aligned}
& \frac{1}{1+|\lambda-g|^{2}} \\
& =\frac{1}{1+|\lambda-\tilde{g}|^{2}} \frac{1+|\lambda-\tilde{g}|^{2}}{1+|\lambda-g|^{2}} \leq \frac{1}{1+|\lambda-\tilde{g}|^{2}} \frac{1+(|\lambda-g|+|g-\tilde{g}|)^{2}}{1+|\lambda-g|^{2}} \\
& \leq \frac{1}{1+|\lambda-\tilde{g}|^{2}} \frac{1+|\lambda-g|^{2}(1+g-\tilde{g})^{2}}{1+|\lambda-g|^{2}}=\frac{(1+g-\tilde{g})^{2}}{1+|\lambda-\tilde{g}|^{2}} .
\end{aligned}
$$

Let $J_{1}:=\left\{n \in \mathbb{N} \mid \lambda_{n} \in \sigma_{g}^{+}(A)\right.$ and $\left.\left|\lambda_{n}-g\right| \geq 1\right\}$ and $J_{2}:=\left\{n \in \mathbb{N} \mid \lambda_{n} \in\right.$ $\sigma_{g}^{+}(A)$ and $\left.\left|\lambda_{n}-g\right|<1\right\}$. Since $J_{2}$ consists only of finitely many points, we get

$$
\sum_{n \in J_{2}} \frac{k_{n}}{\left|\lambda_{n}-g\right|^{2}}<\infty
$$

Thus the statement follows directly from the calculation

$$
\begin{aligned}
& \sum_{n \in J_{1}} \frac{k_{n}}{\left|\lambda_{n}-g\right|^{2}} \\
& \leq 2 \sum_{n \in J_{1}} \frac{k_{n}}{1+\left|\lambda_{n}-g\right|^{2}} \\
& =\frac{2}{g-\tilde{g}}\left[\sum_{n \in J_{1}} k_{n} \frac{\operatorname{Re}\left(\lambda_{n}-\tilde{g}\right)}{1+\left|\lambda_{n}-g\right|^{2}}-\sum_{n \in J_{1}} k_{n} \frac{\operatorname{Re}\left(\lambda_{n}-g\right)}{1+\left|\lambda_{n}-g\right|^{2}}\right] \\
& \stackrel{(38)}{\leq} \frac{2(1+g-\tilde{g})^{2}}{g-\tilde{g}} \sum_{n \in J_{1}} k_{n} \frac{\operatorname{Re}\left(\lambda_{n}-\tilde{g}\right)}{1+\left|\lambda_{n}-\tilde{g}\right|^{2}}-\frac{2}{g-\tilde{g}} \sum_{n \in J_{1}} k_{n} \frac{\operatorname{Re}\left(\lambda_{n}-g\right)}{1+\left|\lambda_{n}-g\right|^{2}} \\
& <\infty . \quad \square
\end{aligned}
$$


The next proposition gives a simple necessary condition for optimizability of a system $\Sigma(A, B)$.

Proposition 6.3. Assume that the system $\Sigma(A, B)$ is optimizable, and let $F$ be an LQ-stabilizing feedback. Then for every $g>g_{b}^{F}$ there exist constants $M_{1}, M_{2}>0$ such that

$$
M_{1} \leq\left\|B^{*} \psi\right\| \leq M_{2}
$$

for every $\psi \in N\left(\bar{\lambda} I-A^{*}\right)$ with $\|\psi\|=1$, where $\lambda$ is an eigenvalue of $A$ with $\operatorname{Re}(\lambda)>g$.

Proof. Let $g>g_{b}^{F}$. Using Proposition 3.6 there exists $M_{1}^{\prime}>0$, such that for every $\psi \in H$ with $\|\psi\|=1$ and every $\lambda \in \mathbb{C}_{g}^{+}$there is a $\xi_{\psi} \in H$ and $\omega_{\psi} \in \mathbb{C}^{m}$ such that

$$
\psi=(\lambda I-A) \xi_{\psi}-B \omega_{\psi}
$$

and $\left\|\xi_{\psi}\right\|_{H},\left\|\omega_{\psi}\right\| \leq M_{1}^{\prime}$. We now choose $\psi \in N\left(\bar{\lambda} I-A^{*}\right)$ with $\|\psi\|=1$, where $\lambda$ is an eigenvalue of $A$ with $\operatorname{Re}(\lambda)>g$. Note that by Lemma 6.2 there exists such a $\psi$. Taking the inner product of (39) with $\psi$, we obtain

$$
1=\left\langle\xi_{\psi},\left(\bar{\lambda} I-A^{*}\right) \psi\right\rangle-\left\langle B \omega_{\psi}, \psi\right\rangle=-\left\langle\omega_{\psi}, B^{*} \psi\right\rangle
$$

and thus

$$
1 \leq M_{1}^{\prime}\left\|B^{*} \psi\right\|
$$

Next we prove that there exists a constant $M_{2}>0$ such that $\left\|B^{*} \psi\right\| \leq M_{2}$. Since $B$ is an admissible control operator for $T(t)$, there exist $t_{1}>0$ and $M>0$ such that

$$
\left\|\int_{0}^{t_{1}} T\left(t_{1}-\rho\right) B u(\rho) d \rho\right\| \leq M\|u\|_{\boldsymbol{L}_{2}\left(0, t_{1} ; \mathbb{C}^{m}\right)}
$$

for every $u \in \boldsymbol{L}_{2}\left(0, t_{1} ; \mathbb{C}^{m}\right)$. Let $K:=\sup _{\lambda \in \sigma(A)} \operatorname{Re}(\lambda)<\infty$ and define

$$
f(\mu):= \begin{cases}\frac{1-e^{-2 \mu t_{1}}}{2 \mu}, & \mu \in[g, K] \backslash\{0\}, \\ t_{1}, & \mu=0 .\end{cases}
$$

By l'Hôspital's rule, $f$ is continuous on $[g, K]$ and so

$$
\sup _{\mu \in[g, K]}|f(\mu)|<\infty \text {. }
$$

Let $\psi \in N\left(\bar{\lambda} I-A^{*}\right)$ with $\|\psi\|=1$, where $\lambda$ is an eigenvalue of $A$ with $\operatorname{Re}(\lambda)>g$. We define $u_{\lambda} \in \boldsymbol{L}_{2}\left(0, t_{1} ; \mathbb{C}\right)$ by

$$
u_{\lambda}(\rho):=\frac{1}{t_{1}} e^{-\lambda\left(t_{1}-\rho\right)}, \quad \rho \in\left[0, t_{1}\right] .
$$

This implies

$$
\begin{aligned}
\left\|B^{*} \psi\right\| & =\sup _{\|u\|=1}\left|\left\langle u, B^{*} \psi\right\rangle\right|=\sup _{\|u\|=1}|\langle B u, \psi\rangle|=\sup _{\|u\|=1}\left|\int_{0}^{t_{1}} u_{\lambda}(\rho) e^{\lambda\left(t_{1}-\rho\right)} d \rho\langle B u, \psi\rangle\right| \\
& =\sup _{\|u\|=1}\left|\int_{0}^{t_{1}}\left\langle B u u_{\lambda}(\rho), e^{\bar{\lambda}\left(t_{1}-\rho\right)} \psi\right\rangle d \rho\right|=\sup _{\|u\|=1}\left|\int_{0}^{t_{1}}\left\langle B u u_{\lambda}(\rho), T^{*}\left(t_{1}-\rho\right) \psi\right\rangle d \rho\right|
\end{aligned}
$$




$$
\begin{aligned}
& =\sup _{\|u\|=1}\left|\int_{0}^{t_{1}}\left\langle T\left(t_{1}-\rho\right) \operatorname{Buu}_{\lambda}(\rho), \psi\right\rangle d \rho\right|=\sup _{\|u\|=1}\left|\left\langle\int_{0}^{t_{1}} T\left(t_{1}-\rho\right) B u u_{\lambda}(\rho) d \rho, \psi\right\rangle\right| \\
& \leq \sup _{\|u\|=1}\left\|\int_{0}^{t_{1}} T\left(t_{1}-\rho\right) B u u_{\lambda}(\rho) d \rho\right\| \leq \sup _{\|u\|=1} M\left\|u u_{\lambda}\right\|_{L_{2}\left(0, t_{1} ; \mathbb{C}^{m}\right)} \\
& \leq \frac{M}{t_{1}} f(\operatorname{Re}(\lambda))^{1 / 2} \leq \frac{M}{t_{1}} \sup _{\mu \in[g, K]}|f(\mu)|^{1 / 2}:=M_{2} .
\end{aligned}
$$

7. More information on the spaces $\boldsymbol{H}_{\boldsymbol{u}}$ and $\boldsymbol{H}_{\boldsymbol{s}}$. In Theorem 5.3 we saw that every optimizable system $\Sigma(A, B)$ with $A$ satisfying SDA can be split into an exponentially stable part and an exactly controllable part. We now give a precise description of the spaces $H_{u}$ and $H_{s}$ appearing in Theorem 5.3. Note that by the definition of $H_{u}$ and $H_{s}$ we have $H=H_{u} \oplus H_{s}$. The main result of this section is the following theorem.

TheOrem 7.1. Suppose $\Sigma(A, B)$ is optimizable and let $A$ satisfy the SDA. Then the spaces $H_{u}$ and $H_{s}$ in Theorem 5.3 are given by

$$
\begin{aligned}
H_{u} & =\overline{\operatorname{span}_{n \in \mathbb{N}} P_{\lambda_{n}} H}, \\
H_{s} & =\left\{x \in H \mid T(\cdot) x \in \boldsymbol{L}_{2}(0, \infty ; H)\right\},
\end{aligned}
$$

where $\sigma(A) \cap \mathbb{C}_{0}^{+}=\left\{\lambda_{n}\right\}_{n \in \mathbb{N}}$ (see Theorem 6.1).

In order to prove this theorem we need a series of lemmas.

Lemma 7.2. Suppose that $\Sigma(A, B)$ is optimizable and let $A$ satisfy the SDA. Then there exists a constant $C>0$ such that

$$
\left\|(\cdot I-A)^{-1} x\right\|_{L_{2}(i \mathbb{R} ; H)} \leq C\|x\|
$$

for every $x \in H$.

Proof. Let $F$ be an LQ-stabilizing feedback, and choose a negative $\sigma$ larger than $g_{b}^{F}$. Then by Proposition 3.6 there exists a constant $\tilde{C}>0$ such that for every $x \in H$ there exist $\xi_{x} \in \boldsymbol{H}_{2}\left(\mathbb{C}_{\sigma}^{+}, H\right)$ and $\omega_{x} \in \boldsymbol{H}_{2}\left(\mathbb{C}_{\sigma}^{+}, \mathbb{C}^{m}\right)$ such that

$$
(i t I-A)^{-1} x=\xi_{x}(i t)-(i t I-A)^{-1} B \omega_{x}(i t), \quad t \in \mathbb{R},
$$

$\left\|\xi_{x}\right\|_{\boldsymbol{H}_{2}\left(\mathbb{C}_{\sigma}^{+}, H\right)} \leq \tilde{C}\|x\|_{H}$ and $\left\|\omega_{x}\right\|_{\boldsymbol{H}_{2}\left(\mathbb{C}_{\sigma}^{+}, \mathbb{C}^{m}\right)} \leq \tilde{C}\|x\|_{H}$. Using Corollary 5.13, we obtain $(\cdot I-A)^{-1} B \in \boldsymbol{L}_{\infty}\left(i \mathbb{R} ; \mathcal{L}\left(\mathbb{C}^{m}, H\right)\right)$ and thus for $x \in H$ (40) implies

$$
\begin{aligned}
& \left\|(\cdot I-A)^{-1} x\right\|_{\boldsymbol{L}_{2}(i \mathbb{R} ; H)} \\
& \leq\left\|\xi_{x}(\cdot)\right\|_{\boldsymbol{L}_{2}(i \mathbb{R} ; H)}+\left\|(\cdot I-A)^{-1} B\right\|_{\boldsymbol{L}_{\infty}(i \mathbb{R} ; \mathcal{L}(U, H))}\left\|\omega_{x}(\cdot)\right\|_{\boldsymbol{L}_{2}\left(i \mathbb{R} ; \mathbb{C}^{m}\right)} \\
& \leq C\|x\|_{H}
\end{aligned}
$$

for some $C>0$ independent of $x$.

Lemma 7.3. Suppose that $\Sigma(A, B)$ is exactly controllable in finite time and that $A$ generates a $C_{0}$-group $T(t)$ on $H$. Then

$$
H=\overline{\operatorname{span}_{n \in \mathbb{N}} P_{\lambda_{n}} H},
$$

where $\lambda_{n}$ are the eigenvalues of $A$.

Proof. For a $C_{0}$-group there is a real constant $\gamma$ such that $-A+\gamma I$ generates an exponentially stable $C_{0}$-semigroup. Since the assumptions made in the theorem also hold for $A-\gamma I$, we may without loss of generality assume that $-A$ generates 
an exponentially stable $C_{0}$-semigroup. By Theorem 3.10 we have that the spectrum of $A$ is purely point spectrum without (finite) accumulation points. We denote this spectrum by $\left\{\lambda_{n}\right\}$.

Define $V:=\overline{\operatorname{span}}_{n \in \mathbb{N}} P_{\lambda_{n}} H$ and $W:=V^{\perp}$, and assume that $W \neq \emptyset$. It is easy to see that $V$ is $T(t)$-invariant. By Lemmas 4.2 and 4.3 we obtain that the system $\Sigma\left(A_{W}, B_{W}\right)$ is exactly controllable in finite time. The corresponding $C_{0}$-semigroup on $W$ is given by

$$
T_{W}(t) x=P_{W} T(t) x, \quad x \in W .
$$

Since $W$ is the orthogonal complement of $V$, it follows that $W$ is $T(t)^{*}$-invariant, and

$$
T_{W}(t)^{*} x=T(t)^{*} x, \quad x \in W .
$$

The generator of $T_{W}(t)$ is given by $A_{W}^{*}:=\left.A^{*}\right|_{W}$. From the fact that the spectrum of $A$ consists of only point spectrum with finite multiplicity and without an accumulation point, it follows that $\sigma\left(A^{*}\right)=\left\{\bar{\lambda}_{n}\right\}_{n}$, and $\rho_{\infty}\left(A^{*}\right)=\rho\left(A^{*}\right)$. Using part (1) of Lemma 4.2 and (41), we have that

$$
\sigma\left(A_{W}^{*}\right) \subset \sigma\left(A^{*}\right)=\left\{\bar{\lambda}_{n}\right\}_{n} .
$$

Let $\varepsilon>0$ be arbitrary. If $\sigma_{-\varepsilon}^{+}\left(A_{W}^{*}\right) \neq \emptyset$, then there exist a $x \in W$ and an $n \in \mathbb{N}$ such that $\left(\cdot I-A_{W}^{*}\right)^{-1} x$ is not holomorphic at $\bar{\lambda}_{n}\left[18\right.$, p. 174]. Since $\left(s I-A_{W}^{*}\right)^{-1} x=$ $\left(s I-A^{*}\right)^{-1} x, s \in \rho\left(A^{*}\right)$, we get $\left(\cdot I-A^{*}\right)^{-1} x$ is not holomorphic at $\bar{\lambda}_{n}$. This implies that

$$
\int_{\Gamma}\left(s I-A^{*}\right)^{-1} x d s \neq 0,
$$

where $\Gamma$ is a simple closed contour in $\mathbb{C}$ with only one point of $\sigma\left(A^{*}\right)$ in its interior, namely $\bar{\lambda}_{n}$, and no points of $\sigma\left(A^{*}\right)$ on $\Gamma$. Now there is a $y \in H$ such that

$$
\left\langle P_{\lambda_{n}} y, x\right\rangle=\left\langle y, \int_{\Gamma}\left(s I-A^{*}\right)^{-1} x d s\right\rangle \neq 0 .
$$

This is in contradiction with $P_{\lambda_{n}} y \in V$ and $x \in W=V^{\perp}$. Thus $\sigma_{-\varepsilon}^{+}\left(A_{W}^{*}\right)=\emptyset$, and therefore $\sigma_{-\varepsilon}^{+}\left(A_{W}\right)=\emptyset$. Since $\Sigma\left(A_{W}, B_{W}\right)$ is exactly controllable in finite time it is LQ-stabilizable as well. Thus Theorem 5.14 shows that there is a constant $M>0$ such that

$$
\left\|T_{W}(t)\right\| \leq M, \quad t \geq 0 .
$$

This implies

$$
\|T(-t)\| \geq\left\|T_{W}(-t)\right\| \geq \frac{1}{\left\|T_{W}(t)\right\|} \geq M^{-1}, \quad t \geq 0
$$

which is in contradiction with the exponential stability of $-A$. Thus the assumption $W \neq \emptyset$ does not hold, and so we get $H=\overline{\operatorname{span}_{n \in \mathbb{N}} P_{\lambda_{n}} H}$.

Since $\boldsymbol{H}_{2}\left(\mathbb{C}_{0}^{+} ; H\right)$ is a closed subset of $\boldsymbol{L}_{2}(i \mathbb{R} ; H)$, we can write

$$
\boldsymbol{L}_{2}(i \mathbb{R} ; H)=\boldsymbol{H}_{2}\left(\mathbb{C}_{0}^{+} ; H\right) \oplus \boldsymbol{H}_{2}\left(\mathbb{C}_{0}^{+} ; H\right)^{\perp},
$$


where $\boldsymbol{H}_{2}\left(\mathbb{C}_{0}^{+} ; H\right)^{\perp}$ is the orthogonal complement in $\boldsymbol{L}_{2}(i \mathbb{R} ; H)$ of $\boldsymbol{H}_{2}\left(\mathbb{C}_{0}^{+} ; H\right)$. In $[3$, Theorem A.6.22] it is shown that

$$
\boldsymbol{H}_{2}\left(\mathbb{C}_{0}^{+} ; H\right)^{\perp}=\left\{f: \mathbb{C}_{0}^{-} \rightarrow H \mid f(-\cdot) \in \boldsymbol{H}_{2}\left(\mathbb{C}_{0}^{+} ; H\right)\right\} .
$$

Proof of Theorem 7.1. By Theorem 5.3 there exists a projection $P \in \mathcal{L}(H)$ such that (1)-(5) of Theorem 5.3 are satisfied. Lemma 7.2 shows that $(\cdot I-A)^{-1} x \in$ $\boldsymbol{L}_{2}(i \mathbb{R} ; H)$ for every $x \in H$. If we define

$$
\begin{aligned}
& \tilde{H}_{s}=\left\{x \in H \mid(\cdot I-A)^{-1} x \in \boldsymbol{H}_{2}\left(\mathbb{C}_{0}^{+} ; H\right)\right\} \text { and } \\
& \tilde{H}_{u}=\left\{x \in H \mid(\cdot I-A)^{-1} x \in \boldsymbol{H}_{2}\left(\mathbb{C}_{0}^{+} ; H\right)^{\perp}\right\},
\end{aligned}
$$

then (42) shows that

$$
\tilde{H}_{s} \cap \tilde{H}_{u}=\{0\}
$$

1. We now prove that $\tilde{H}_{s}$ and $\tilde{H}_{u}$ are closed, linear subspaces of $H$. We will prove this result only for $\tilde{H}_{s}$, as the proof for $\tilde{H}_{u}$ is very similar. That $\tilde{H}_{s}$ is a linear subspace of $H$ follows immediately from the fact that $\boldsymbol{H}_{2}\left(\mathbb{C}_{0}^{+}, H\right)$ is a linear vector space. In order to prove the closedness we choose a sequence $\left\{z_{n}\right\}_{n}$ in $\tilde{H}_{s}$ which converges to $z \in H$. By Lemma 7.2 we get

$$
\lim _{n \rightarrow \infty}\left\|(\cdot I-A)^{-1} z_{n}-(\cdot I-A)^{-1} z\right\|_{\boldsymbol{L}_{2}(i \mathbb{R}, H)} \leq C \lim _{n \rightarrow \infty}\left\|z_{n}-z\right\|_{H}=0 .
$$

Since $\boldsymbol{H}_{2}\left(\mathbb{C}_{0}^{+} ; H\right)$ is a closed subspace of $\boldsymbol{L}_{2}(i \mathbb{R}, H)$, this implies that $(\cdot I-$ $A)^{-1} z \in \boldsymbol{H}_{2}\left(\mathbb{C}_{0}^{+} ; H\right)$, and so $\tilde{H}_{s}$ is closed.

2. We now prove that $H_{u} \subset \tilde{H}_{u}$. Since $\sigma_{g_{b}^{F}}^{+}(A)$ consists only of point spectrum with no (finite) accumulation point, we have that

$$
\rho_{\infty}(A) \cap \mathbb{C}_{g_{b}^{F}}^{+}=\rho(A) \cap \mathbb{C}_{g_{b}^{F}}^{+} .
$$

Since the imaginary axis is in the resolvent set, by Lemma 4.2 we have for all $t \in \mathbb{R}$ and $x \in H_{u}$

$$
(i t I-A)^{-1} x=\left(i t I-A_{u}\right)^{-1} .
$$

The operator $-A_{u}$ generates an exponentially stable semigroup on $H_{u}$, and hence for $x \in H_{u}$ we get that $\left(i t I-A_{u}\right)^{-1} x$ is the Fourier transform of $-T_{u}(t) x, t \in(-\infty, 0)$. This last function is in $\boldsymbol{L}_{2}(-\infty, 0 ; H)$. By the PaleyWiener theorem [3, Theorem A.6.21], (43), and (44), this implies that $(\cdot I-$ $A)^{-1} x \in \boldsymbol{H}_{2}\left(\mathbb{C}_{0}^{+} ; H\right)^{\perp}$, and thus $x \in \tilde{H}_{u}$.

3. We now prove that $H_{s} \subset \tilde{H}_{s}$. Let $x \in H_{s}$ arbitrary. Then $T(\cdot) x \in \boldsymbol{L}_{2}(0, \infty ; H)$ and thus the Paley-Wiener theorem [3, Theorem A.6.21] implies that its Laplace transform $(\cdot I-A)^{-1} x$ is in $\boldsymbol{H}_{2}\left(\mathbb{C}_{0}^{+} ; H\right)$. Thus $x \in \tilde{H}_{s}$.

4. We now prove $H_{u}=\tilde{H}_{u}$ and $H_{s}=\tilde{H}_{s}$. We prove this result only for $\tilde{H}_{s}$, as the proof for $\tilde{H}_{u}$ is very similar. Assume that $H_{s}=\tilde{H}_{s}$ does not hold. Then there exists an element $x \in \tilde{H}_{s} \backslash H_{s}$. We can write $x$ as $x=x_{u}+x_{s}$ with $x_{u} \in H_{u}$ and $x_{s} \in H_{s}$. Since $x_{u}=x-x_{s} \in \tilde{H}_{s}$, we obtain $x_{u} \in H_{u} \cap \tilde{H}_{s} \subset \tilde{H}_{u} \cap \tilde{H}_{s}=\{0\}$, which is in contradiction with $x \in \tilde{H}_{s} \backslash H_{s}$. 
5. We now prove that $V \subset H_{u}$, where

$$
V:=\overline{\operatorname{span}_{n \in \mathbb{N}} P_{\lambda_{n}} H},
$$

and $\left\{\lambda_{n}\right\}_{n}=\sigma(A) \cap \mathbb{C}_{0}^{+}$. First of all we choose $x \in P_{\lambda_{n}} H$ for some $n \in \mathbb{N}$. Then in [3, p. 99] it has been proven that

$$
(i t I-A)^{-1} x=\sum_{j=0}^{m_{a}\left(\lambda_{n}, A\right)}(-1)^{j} \frac{\left(\lambda_{n} I-A\right)^{j} x}{\left(i t-\lambda_{n}\right)^{j+1}}, \quad t \in \mathbb{R},
$$

where $m_{a}\left(\lambda_{n}, A\right)$ is the algebraic multiplicity of the eigenvalue $\lambda_{n}$. Since $\operatorname{Re}\left(\lambda_{n}\right)>0$, we have

$$
\frac{1}{\left(\cdot-\lambda_{n}\right)^{j}} \in \boldsymbol{H}_{2}\left(\mathbb{C}_{0}^{+} ; H\right)^{\perp}
$$

and so we obtain that $x \in \tilde{H}_{u}$. Now $H_{u}=\tilde{H}_{u}$ and $V$ are closed, linear subspaces of $H$ and thus the statement is proved.

6. In order to prove the theorem it now remains to show that $V=H_{u}$ holds. The system $\Sigma\left(A_{u},(I-\tilde{P}) B\right)$ is exactly controllable in finite time. Thus Lemma 7.3 proves $V=H_{u}$.

8. An example. We now construct an example that is optimizable, but it is not possible to split the state space in a direct sum as given in Theorem 1.3. The generator in this example does not satisfy the SDA at any negative number. For the construction of this example the following lemmas will be useful. For background information on Carleson measures we refer to Garnett [9, p. 31].

Lemma 8.1. Let $\left\{q_{n}\right\}_{n \in \mathbb{N}}=\mathbb{Q} \cap\left(\frac{1}{2}, \infty\right)$ be chosen such that

$$
q_{k+\frac{l(l-1)}{2}} \in[k-1, k], \quad l \in \mathbb{N}, k \in\{1, \ldots, l\},
$$

and define $\left\{\gamma_{n}\right\}_{n \in \mathbb{N}} \subset \mathbb{C}_{0}^{+}$by

$$
\gamma_{n}:=q_{n}+i n^{4}, \quad n \in \mathbb{N} .
$$

Then $\mu:=\sum_{n \in \mathbb{N}} \operatorname{Re} \gamma_{n} \delta_{\gamma_{n}}$ is a Carleson measure.

Proof. In order to prove this we define

$$
S\left(h, y_{0}\right):=\left\{z \in \mathbb{C}_{0}^{+} \mid 0<\operatorname{Re} z<h, y_{0}<\operatorname{Im} z<y_{0}+h\right\}, \quad h>0, y_{0} \in \mathbb{R} .
$$

Now $\mu$ is a Carleson measure if and only if there exists a constant $A>0$ such that $\mu\left(S\left(h, y_{0}\right)\right) \leq A h$ for every $h>0$ and all $y_{0} \in \mathbb{R}$ (see, for example, $[9$, p. 31]).

Clearly, $\mu\left(S\left(h, y_{0}\right)\right)=0$ if $h \in\left[0, \frac{1}{2}\right]$. For $h>\frac{1}{2}$ and $y_{0}<-h$, we have that $\mu\left(S\left(h, y_{0}\right)\right)=0$ as well.

We now consider the situation $h>\frac{1}{2}$ and $y_{0} \geq h^{2}$. Then for every $n \in \mathbb{N}$ with $y_{0}<n^{4}<y_{0}+h$, we get

$$
(n+1)^{4}>n^{4}+n^{2}>y_{0}+\sqrt{y_{0}} \geq y_{0}+h .
$$

Thus $\#\left\{\left\{\gamma_{n}\right\}_{n \in \mathbb{N}} \cap S\left(h, y_{0}\right)\right\} \leq 1$. Combining this with the fact that if $\gamma_{n} \in S\left(h, y_{0}\right)$, then $q_{n}$ is less than $h$, shows that $\mu\left(S\left(h, y_{0}\right)\right) \leq h$. 
Finally, we have to consider the situation $h>\frac{1}{2}, y_{0} \in\left(-h, h^{2}\right)$. Then

$$
S\left(h, y_{0}\right) \subset R(h):=\left\{z \in \mathbb{C}_{0}^{+} \mid 0<\operatorname{Re} z<h,-h<\operatorname{Im} z<h^{2}+h\right\} .
$$

Using $h>\frac{1}{2}$, we see that

$$
h^{2}+h \leq h^{2}+2 h^{2}<16 h^{2} .
$$

From this we get $\#\left\{\left\{\gamma_{n}\right\}_{n \in \mathbb{N}} \cap R(h)\right\} \leq 2 \sqrt{h}$. We now choose $l_{h} \in \mathbb{N}$ such that

$$
\frac{l_{h}\left(l_{h}-1\right)}{2}<2 \sqrt{h}<\frac{l_{h}\left(l_{h}+1\right)}{2} .
$$

This implies immediately

$$
l_{h} \leq 1+4 h^{1 / 4}<6 h^{1 / 4},
$$

where we have used that $h>\frac{1}{2}$. Thus

$$
\mu\left(S\left(h, y_{0}\right)\right) \leq \mu(R(h)) \leq \sum_{n=1}^{[2 \sqrt{h}]} q_{n} \leq \sum_{l=1}^{l_{h}} \sum_{k=1}^{l} k=\frac{1}{6} l_{h}\left(l_{h}+1\right)\left(l_{h}+2\right) \leq A h^{3 / 4},
$$

where $A>0$ is independent of $h$. Thus $\mu$ is a Carleson measure.

Lemma 8.2. Let $\left\{q_{n}\right\}_{n \in \mathbb{N}}=\mathbb{Q} \cap\left(\frac{1}{2}, \infty\right)$ be chosen as in Lemma 8.1 and define $\left\{\beta_{n}\right\}_{n \in \mathbb{N}}$ by

$$
\beta_{2 n}:=\gamma_{n}, \quad \beta_{2 n-1}:=\gamma_{n}+\frac{1}{n}, \quad n \in \mathbb{N} .
$$

Moreover, let $\Theta \in \boldsymbol{H}_{\infty}\left(\mathbb{C}_{0}^{+}\right)$be the Blaschke product corresponding to the zeros $\left\{\beta_{n}\right\}$, i.e.,

$$
\Theta(s):=\prod_{n=1}^{\infty} \frac{\left|1-\beta_{n}^{2}\right|}{1-\beta_{n}^{2}} \frac{s-\beta_{n}}{s+\bar{\beta}_{n}}, \quad s \in \mathbb{C}_{0}^{+} .
$$

Then

$$
\sup _{0<\operatorname{Res}<1 / 4}\left|\Theta^{-1}(s)\right|<\infty .
$$

Proof. For $x \in\left(0, \frac{1}{4}\right), y \in \mathbb{R}$, we get

$$
\begin{aligned}
\left|\Theta(x+i y)^{-2}\right| & =\prod_{n \in \mathbb{N}} \frac{\left(x+\operatorname{Re} \beta_{n}\right)^{2}+\left(y-\operatorname{Im} \beta_{n}\right)^{2}}{\left(x-\operatorname{Re} \beta_{n}\right)^{2}+\left(y-\operatorname{Im} \beta_{n}\right)^{2}} \\
& =\prod_{n \in \mathbb{N}}\left(1+\frac{4 x \operatorname{Re} \beta_{n}}{\left(x-\operatorname{Re} \beta_{n}\right)^{2}+\left(y-\operatorname{Im} \beta_{n}\right)^{2}}\right) .
\end{aligned}
$$

Thus (45) is equivalent to

$$
\sup _{x \in\left(0, \frac{1}{4}\right)} \sup _{y \in \mathbb{R}} \sum_{n \in \mathbb{N}} \ln \left(1+\frac{4 x \operatorname{Re} \beta_{n}}{\left(x-\operatorname{Re} \beta_{n}\right)^{2}+\left(y-\operatorname{Im} \beta_{n}\right)^{2}}\right)<\infty .
$$


Using $\ln (1+z) \leq z, z \geq 0$, and

$$
0 \leq \frac{4 x \operatorname{Re} \beta_{n}}{\left(x-\operatorname{Re} \beta_{n}\right)^{2}+\left(y-\operatorname{Im} \beta_{n}\right)^{2}},
$$

it is easy to see that

$$
\sup _{x \in\left(0, \frac{1}{4}\right)} \sup _{y \in \mathbb{R}} \sum_{n \in \mathbb{N}} \frac{x \operatorname{Re} \beta_{n}}{\left(x-\operatorname{Re} \beta_{n}\right)^{2}+\left(y-\operatorname{Im} \beta_{n}\right)^{2}}<\infty
$$

implies (46). Thus it remains to prove that

$$
h(x, y):=\sum_{n \in \mathbb{N}} \frac{x \operatorname{Re} \beta_{n}}{\left(x-\operatorname{Re} \beta_{n}\right)^{2}+\left(y-\operatorname{Im} \beta_{n}\right)^{2}}
$$

is uniformly bounded on $\mathbb{C}_{0}^{+} \cap \mathbb{C}_{1 / 4}^{-}$.

Let $x+i y \in \mathbb{C}_{0}^{+} \cap \mathbb{C}_{1 / 4}^{-}$be arbitrary. Define $z:=\frac{1}{3}-x$. From $\operatorname{Re} \beta_{n}>\frac{1}{2}$, we get

$$
\begin{aligned}
h(x, y) & =\sum_{n \in \mathbb{N}} \frac{\left(\frac{1}{3}-z\right) \operatorname{Re} \beta_{n}}{\left(\frac{1}{3}-z-\operatorname{Re} \beta_{n}\right)^{2}+\left(y-\operatorname{Im} \beta_{n}\right)^{2}} \\
& \leq 3 \sum_{n \in \mathbb{N}} \frac{\left(\frac{1}{3}-z\right)\left(\operatorname{Re} \beta_{n}-\frac{1}{3}\right)}{\left(\frac{1}{3}-z-\operatorname{Re} \beta_{n}\right)^{2}+\left(y-\operatorname{Im} \beta_{n}\right)^{2}} \\
& \leq 3 \sum_{n \in \mathbb{N}} \operatorname{Re} \gamma_{n}\left|g_{z, y}\left(\gamma_{n}\right)\right|^{2},
\end{aligned}
$$

where $\gamma_{n}:=\beta_{n}-\frac{1}{3} \in \mathbb{C}_{0}^{+}$and

$$
g_{z, y}(s)=\frac{\sqrt{\frac{1}{3}-z}}{s+z-i y} \in \boldsymbol{H}_{2}\left(\mathbb{C}_{0}^{+}\right) .
$$

By Lemma $8.1 \sum_{n \in \mathbb{N}} \operatorname{Re} \beta_{2 n} \delta_{\beta_{2 n}}$ is a Carleson measure. Similarly, it can be shown that $\sum_{n \in \mathbb{N}} \operatorname{Re} \beta_{2 n+1} \delta_{\beta_{2 n+1}}$ is a Carleson measure. From this it follows easily that $\sum_{n \in \mathbb{N}} \operatorname{Re} \gamma_{n} \delta_{\gamma_{n}}$ also is a Carleson measure. Hence, Avdonin and Ivanov [2, p. 56] or Garnett [9, Theorem 3.9] implies

$$
h(x, y) \leq C\left\|g_{z, y}\right\|_{\boldsymbol{H}_{2}\left(\mathbb{C}_{0}^{+}\right)}^{2}=C\left\|e^{-(z-i y) \cdot} \sqrt{\frac{1}{3}-z}\right\|_{\boldsymbol{L}_{2}(0, \infty)}^{2}=\frac{C}{2} \frac{x}{\frac{1}{3}-x} \leq \frac{3 C}{2} .
$$

Thus $\left|\Theta(s)^{-1}\right|$ is uniformly bounded on $\mathbb{C}_{0}^{+} \cap \mathbb{C}_{1 / 4}^{-}$.

The following realization result can be found in Salamon [30] and in Ober and $\mathrm{Wu}$ [21]. The proof in the notation of this paper is documented in Jacob and Zwart [16].

Lemma 8.3. Let $\Theta$ be given as in Lemma 8.2. Then there exist a Hilbert space $V$, an exponentially stable $C_{0}$-semigroup $T(t)$ on $V$ with generator $A$, and an admissible control operator $B$ for $T(t)$ such that the system $\Sigma(A, B)$ is exactly controllable in finite time, every $-\bar{\beta}_{n}, n \in \mathbb{N}$, is an eigenvalue of $A$, and the function $x_{n}(s):=\frac{1}{s+\bar{\beta}_{n}}$ is a corresponding eigenvector. 
EXAmple 8.4. We now consider the system $\Sigma(A, B)$ given as in Lemma 8.3. Since $\Sigma(A, B)$ is exactly controllable in finite time, by Lemma 3.8 and Proposition 3.9 the system $\Sigma(A+I, B)$ is optimizable. Lemma 8.3 shows that $\left\{\lambda_{n}\right\}_{n \in \mathbb{N}} \subseteq \sigma(A+I)$ holds, where $\lambda_{n}:=-\bar{\beta}_{n}+1$, and $x_{\lambda_{n}}(s):=\frac{1}{s-\lambda_{n}}$ is an eigenvector corresponding to the eigenvalue $\lambda_{n}$. Thus the spectrum of $A+I$ does not satisfy the SDA at any negative number.

Next, we show that it is not possible to decompose the state space $V$ as $V=$ $V_{s} \oplus V_{u}$, where

$$
\begin{aligned}
V_{s} & :=\left\{x_{0} \in V \mid e^{\left.-g \cdot T(\cdot) x_{0} \in \boldsymbol{L}_{2}(0, \infty ; V)\right\},} \underset{\lambda \in \sigma(A+I) \text { with } \operatorname{Re}(\lambda) \geq g}{P_{\lambda} V}\right.
\end{aligned}
$$

for some $g<0$. Let $g<0$ be arbitrary. It is easy to see that $x_{\lambda_{n}} \in V_{u}$ if $\operatorname{Re}\left(\lambda_{n}\right) \geq g$. Next, we show that $x_{\lambda_{n}} \in V_{s}$ holds if $\operatorname{Re}\left(\lambda_{n}\right)<g$. Let $n \in \mathbb{N}$ with $\operatorname{Re}\left(\lambda_{n}\right)<g$ be arbitrary. Since $x_{\lambda_{n}}$ is an eigenvector of $A$ with eigenvalue $\lambda_{n}$, we have that

$$
T(t) x_{\lambda_{n}}=e^{\lambda_{n} t} x_{\lambda_{n}} .
$$

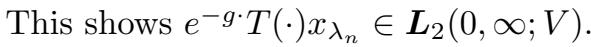

By the construction of the sequence $\left\{\beta_{n}\right\}_{n \in \mathbb{N}}$ there now exist subsequences $\left\{\mu_{n}\right\}_{n \in \mathbb{N}}$, $\left\{\nu_{n}\right\}_{n \in \mathbb{N}} \subseteq\left\{\lambda_{n}\right\}_{n \in \mathbb{N}}$ such that

(1) $\operatorname{Re} \mu_{n}<g<\operatorname{Re} \nu_{n}, n \in \mathbb{N}$,

(2) $\nu_{n}=\mu_{n}+\alpha_{n}, n \in \mathbb{N}$, with $\alpha_{n}>0$ and $\lim _{n \rightarrow \infty} \alpha_{n}=0$,

(3) $\lim _{n \rightarrow \infty} \operatorname{Re} \mu_{n}=g$ and $\lim _{n \rightarrow \infty} \operatorname{Im} \mu_{n}=\infty$.

Thus $x_{\mu_{n}} \in V_{s}$ and $x_{\nu_{n}} \in V_{u}$ for every $n \in \mathbb{N}$. It is now easy to see that

$$
\left\|x_{\nu_{n}}\right\|^{2}=\frac{\pi}{\left|\operatorname{Re} \nu_{n}\right|}, \quad n \in \mathbb{N},
$$

and

$$
\lim _{n \rightarrow \infty}\left\|x_{\mu_{n}}-x_{\nu_{n}}\right\|=0 .
$$

Let us now assume that $V=V_{s} \oplus V_{u}$ is satisfied. Then there would exist a projection $P \in \mathcal{L}(V)$ with $\operatorname{ker} P=V_{s}$ and $\operatorname{Im} P=V_{u}$. Thus

$$
\begin{aligned}
\frac{\pi}{|g|}=\lim _{n \rightarrow \infty}\left\|x_{\nu_{n}}\right\|^{2} & =\lim _{n \rightarrow \infty}\left\|P x_{\nu_{n}}\right\|^{2}=\lim _{n \rightarrow \infty}\left\|P\left(x_{\nu_{n}}-x_{\mu_{n}}\right)\right\|^{2} \\
& \leq\|P\|^{2} \lim _{n \rightarrow \infty}\left\|x_{\nu_{n}}-x_{\mu_{n}}\right\|^{2}=0,
\end{aligned}
$$

which is a contradiction. Hence $V=V_{s} \oplus V_{u}$ is not satisfied.

9. Conclusion. For an infinite-dimensional optimizable system with a finiterank admissible control operator we showed that the system can be decomposed into an exponentially stable subsystem and an exactly controllable subsystem. For the proof we needed the spectrum to be able to be decomposed into a stable and an unstable part. The example in section 8 shows that without this SDA Theorem 1.3 does not hold. Since this example is very technical, we feel that every optimizable system encountered in practice satisfies the SDA. Note that Theorem 1.4 already shows that the unstable part of the spectrum of an optimizable system consists of isolated points. 
We gave characterizations of the state spaces of the subsystems. The state space of the unstable part equals the span of all unstable (generalized) eigenvectors and the state space of the exponentially stable part is given by all vectors for which the action of the original semigroup is stable.

From our results we derived easy necessary conditions for a system to be optimizable; see, e.g., Example 5.7, Theorem 6.1, and Proposition 6.3 or Jacob and Zwart $[15]$.

\section{REFERENCES}

[1] W. Arendt, A. Grabosch, G. Greiner, U. Groh, H. P. Lotz, U. Moustakas, R. Nagel, F. Neubrander, and U. Schlotterbeck, One Parameter Semigroups of Positive Operators, Lecture Notes in Math. 1184, Springer-Verlag, Berlin, 1986.

[2] S. A. Avdonin And S. A. Ivanov, Families of Exponentials: The Method of Moments in Controllability Problems for Distributed Parameter Systems, Cambridge University Press, Cambridge, UK, 1995.

[3] R. F. Curtain and H. Zwart, An Introduction to Infinite-Dimensional Linear Systems Theory, Texts Appl. Math. 21, Springer-Verlag, New York, 1995.

[4] R. DATKo, A linear control problem in abstract Hilbert space, J. Differential Equations, 9 (1971), pp. 346-359.

[5] W. Desch AND W. SChappacher, Spectral properties of finite-dimensional perturbed linear semigroups, J. Differential Equations, 59 (1985), pp. 80-102.

[6] P. L. Duren, Theory of $H^{p}$ Spaces, Pure Appl. Math. 38, Academic Press, San Diego, 1970.

[7] H. O. FatTorini, Exact controllability of linear systems in infinite dimensional spaces, in Partial Differential Equations and Related Topics, Tulane Univ., New Orleans, Lecture Notes in Math. 446, Springer-Verlag, New York, 1975, pp. 166-183.

[8] F. Flandoli, I. Lasiecka, And R. Triggiani, Algebraic Riccati equations with non-smoothing observation arising in hyperbolic and Euler-Bernoulli boundary control problems, Ann. Mat. Pura Appl., CLIII (1988), pp. 307-382.

[9] J. B. Garnett, Bounded Analytic Functions, Academic Press, New York, 1981.

[10] P. Grabowski and F. M. Callier, Admissible observation operators, duality of observation and control, Technical report 94-27, Département de Mathématiques, Facultés Universitaires de Namur, Namur, Belgium, 1994.

[11] G. Greiner, J. Voigt, And M. WolfF, On the spectral bound of the generator of semigroups with positive operators, J. Operator Theory, 5 (1981), pp. 245-256.

[12] M. L. J. Hautus, (A, B)-invariant and stabilizability subspace, a frequency domain description, Automatica J., 16 (1980), pp. 703-707.

[13] E. Hille And R. S. Phillips, Functional Analysis and Semi-Groups, Amer. Math. Soc. Colloq. Publ. 31, AMS, Providence, RI, 1957.

[14] F. Huang, Characteristic conditions for exponential stability of linear dynamical systems in Hilbert spaces, Ann. Differential Equations, 1 (1985), pp. 43-55.

[15] B. Jacob And H. Zwart, On lack of optimizability, in Proceedings of the European Control Conference, Conference ID 976, Timing FR-A-E-3, Brussels, 1997.

[16] B. JaCOB AND H. ZWART, Realization of inner functions, Technical report 1998-17, School of Mathematics, University of Leeds, Leeds, UK, 1998.

[17] C. A. Jacobson And C. N. NetT, Linear state space systems in infinite-dimensional space: The role and characterization of joint stabilizability/detectability, IEEE Trans. Automat. Control, AC-33 (1988), pp. 541-550.

[18] T. Kato, Perturbation Theory for Linear Operators, Classics Math., Springer-Verlag, Berlin, 1995.

[19] M. G. Kurtz, A general theorem on the convergence of operator semigroups, Trans. Amer. Math. Soc., 148 (1980), pp. 23-32.

[20] S. A. Nefedov and F. A. Sholokhovich, A criterion for the stability of dynamical systems with finite-dimensional input, Differentsial'nye Uravneniya, 22 (1986), pp. 163-166.

[21] R. J. OBER AND Y. Wu, Infinite-dimensional continuous-time linear systems: Stability and structure analysis, SIAM J. Control Optim., 34 (1996), pp. 757-812.

[22] A. PAZY, Semigroups of Linear Operators and Applications to Partial Differential Equations, Springer-Verlag, Berlin, 1983.

[23] A. J. Pritchard And J. ZabCZyk, Stability and stabilizability of infinite dimensional systems, SIAM Rev., 23 (1981), pp. 25-52. 
[24] J. Prüss, On the spectrum of $C_{0}$-semigroups, Trans. Amer. Math. Soc., 284 (1984), pp. 847857.

[25] R. REBARBER, Necessary conditions for exponential stability of distributed parameter systems with infinite dimensional unbounded feedback, Systems Control Lett., 14 (1990), pp. 241248.

[26] R. REBARBer, Conditions for the equivalence of internal and external stability for distributed parameter systems, IEEE Trans. Automat. Control, 38 (1993), pp. 994-998.

[27] R. Rebarber and G. Weiss, An extension of Russell's principle on exact controllability, in European Control Conference, Conference ID 736, Timing TH-E-E-2, Brussels, 1997.

[28] R. Rebarber and H. J. Zwart, Open loop stabilizability of infinite-dimensional systems, Math. Control Signals Systems, 11 (1998), pp. 129-160.

[29] D. L. Russell, Controllability and stabilizability theory for linear partial differential equations: Recent progress and open problems, SIAM Rev., 20 (1978), pp. 639-739.

[30] D. Salamon, Realization theory in Hilbert space, Math. Systems Theory, 21 (1989), pp. 147164.

[31] R. Triggiani, On the stabilizability problem in Banach spaces, J. Math. Anal. Appl., 52 (1975), pp. 383-403.

[32] G. WeIss, Admissibility of input elements for diagonal semigroups on $l^{2}$, Systems Control Lett., 10 (1988), pp. 79-82.

[33] G. WeIss, Admissibility of unbounded control operators, SIAM J. Control Optim., 27 (1989), pp. $527-545$.

[34] G. WeIss, Admissible observation operators for linear semigroups, Israel J. Math., 65 (1989), pp. $17-43$.

[35] G. WeIss, Two conjectures on the admissibility of control operators, in Estimation and Control of Distributed Parameter Systems, W. Desch, F. Kappel, and K. Kunisch, eds., Birkhäuser Verlag, Basel, 1991, pp. 367-378.

[36] G. Weiss, Regular linear systems with feedback, Math. Control Signals Systems, 7 (1994), pp. 23-57.

[37] G. Weiss and R. F. Curtain, Dynamical stabilization of regular linear systems, IEEE Trans. Automat. Control, 42 (1997), pp. 4-21.

[38] R. M. Young, An Introduction to Nonharmonic Fourier Series, Academic Press, New York, 1980.

[39] J. ZABCZYK, A note on $C_{0}$-semigroups, Bull. Polish Acad. Sci. Math., 23 (1975), pp. 895-898.

[40] H. J. ZWART, Equivalence between open-loop and closed-loop invariance for infinitedimensional systems: A frequency domain approach, SIAM J. Control Optim., 26 (1988), pp. 1175-1199.

[41] H. J. ZWART, Linear quadratic optimal control for abstract linear systems, in Conference on Modelling and Optimization of Distributed Parameter Systems Applications to Engineering, Warsaw, Poland, Chapman \& Hall, New York, 1996, pp. 175-182 